\title{
The Dependence of the Damping Rate of Medium-n Toroidal Alfvén Eigenmodes on the Edge Plasma Elongation in $\mathrm{JET}^{+}$
}

${ }^{+}$this paper is an extended version of a contribution presented at the $11^{\text {th }}$ IAEA Technical Committee Meeting on Energetic Particles, Kyiv (Ukraine), 21-23 September 2009

D.Testa $^{1}$, N.Mellet ${ }^{1}$, T.Panis ${ }^{1}$, P.Blanchard ${ }^{1,2}$, H.Carfantan ${ }^{3}$, A.Fasoli ${ }^{1}$, JET-EFDA contributors ${ }^{*}$ JET-EFDA, Culham Science Centre, OX14 3DB, Abingdon, UK e-mail address of main author: duccio.testa@epfl.ch

[1] Centre de Recherches en Physique des Plasmas, Ecole Polytechnique Fédérale de Lausanne (CRPP-EPFL), Association EURATOM - Confédération Suisse, CH-1015 Lausanne, Switzerland [2] JET-EFDA Close Support Unit, Culham Science Centre, OX14 3DB, Abingdon, UK [3] Laboratoire d'Astrophysique de Toulouse - Tarbes (LATT), Université de Toulouse - CNRS, 31400 Toulouse, France

\begin{abstract}
$\underline{\text { Abstract }}$
This paper reports the first quantitative analysis of the measurements of the damping rate $(\gamma / \omega)$ for stable Alfvén Eigenmodes (AEs) with toroidal mode number (n) in the range $|n|=3 \div 15$ as function of the edge plasma elongation $\left(\kappa_{95}\right)$. We find that the damping rate $\gamma / \omega$ vs. $\kappa_{95}$ for medium-n Toroidal AEs, with $\mathrm{n}=3$ and $\mathrm{n}=7$, increases for increasing elongation, i.e. its scaling vs. $\kappa_{95}$ follows the same trend previously measured and explained theoretically for the $n=1$ and $n=2$ TAE modes. Theoretical analysis of the measurements for the $n=3$ TAEs has been performed using the LEMan code. The results are in excellent agreement for all the magnetic configurations where there is only a very minor up/down asymmetry in the poloidal cross-section of the plasma. These experimental results further confirm the possibility of using the edge shape parameters as a real-time actuator for control of the stability of alpha-particles driven AEs in burning plasma experiments, such as ITER.
\end{abstract}

PACS Classification scheme: D0, Te.

\footnotetext{
* Appendix of F.Romanelli, “Overview of JET Results”, 22 ${ }^{\text {nd }}$ IAEA Fusion Energy Conference, Geneva, 2008 ; Nucl. Fus. 49 (2009), 104006; weblink: http://iopscience.iop.org/0029-5515/49/10/104006.
} 


\section{Introduction}

One of the most important physics issues on the way to a fusion reactor is the understanding and the control of burning plasmas, the operational regime where the energy carried by the fusion produced alpha particles ( $\alpha s)$ exceeds that which has been externally injected to initiate the thermonuclear fusion process. Burning plasmas are characterized by a very strong coupling between their various operational elements, such as the pressure profile of the background plasma, which drives the fusion reactivity but may also cause the onset of magnetic instabilities, and the distribution in phase space of the fusion-born $\alpha$ s and their interaction with the background coherent and turbulent instability spectrum. The present-day fusion experiments, such as JET, approach this problem by investigating separately some of the individual elements of this regime, namely increasing the fusion gain and controlling the background current and pressure driven magnetic instabilities, before actual burning plasma conditions can be achieved in forthcoming devices such as ITER.

One of these elements is the resonant interaction of the $\alpha$ s with coherent plasma waves that can be produced by the $\alpha$ s themselves due to excessive peaking of their pressure gradient. This interaction can lead to an efficient energy and momentum exchange between the waves and the $\alpha \mathrm{s}[1,2]$. If this mechanism leads to a significant spatial re-distribution of the $\alpha$ s themselves up to the vessel walls, not only will the overall fusion performance be limited, but also the machine integrity may be severely affected because of damage to the first wall.

Among all the different classes of AEs [3], of particular interest are AEs with toroidal mode number (n) in the range $n \sim 3 \div 20$, as these are expected to interact most strongly with the $\alpha s$ [4]. The stability of AEs with these medium-n mode numbers is investigated experimentally in JET using a new set of compact in-vessel antennas [5] which provides a direct measurement of the damping rate $(\gamma / \omega)$ as function of the background plasma parameters for individual toroidal mode numbers. These measurements are now being routinely obtained in JET in different operating scenarios. In this paper we report the first quantitative analysis of the dependence of the damping rate on the edge plasma elongation $\left(\kappa_{95}\right)$, which has prompted some direct comparison with code predictions.

This paper is an extended version of a contribution presented at the $11^{\text {th }}$ IAEA Technical Committee Meeting on energetic particles [6]. As such, this presentation is organised as follows. Section-2 gives a brief description of the new JET AE active antenna system. Section-3 reports the first quantitative measurements of the damping rate for Toroidal AEs (TAEs) with $n=3$ and $n=7$, focussing particularly on the dependence vs. the edge elongation. In Section-4 we present the first simulations that have been run to model the $n=3$ data using the LEMan code $[7,8]$. Finally, in Section-5 we present the conclusion of this work and an outline towards future activities. Two 
paper post IAEA-TCM-EP-2009: accepted for Nuclear Fusion Special Issue EP2010, March 2010

companion papers $[9,10]$ report on some of the technical aspects of the new antenna system and on various additional measurements of the damping rate for medium-n TAEs.

\section{The New Active Antenna System for Excitation of Medium-n Alfvén Eigenmodes}

The measurement of the AE damping rate has been a long-standing feature of the JET experimental program: the first data were collected for low-n TAEs $(n=0,|n|=1,|n|=2)$ since the mid-nineties using the saddle coils system [11]. This system had one main limitation, namely the possibility of driving only modes up to $|\mathrm{n}|=2$ because of the in-vessel geometry of the saddle coils. After many years of successful operation, during which we obtained in excess of $10^{5}$ individual damping rate measurements and performed many different experimental scans, the saddle coils were dismantled during the 2004-2005 shutdown. A new set of in-vessel antennas was designed to overcome the nnumber limitations of the saddle coils, and was finally installed in 2005. In its final implementation, the system comprises two assemblies of four toroidally closely-spaced coils each, situated at opposite toroidal locations. Calculations performed with the LION code [12] demonstrate that this arrangement provides, for the same JET equilibrium, a coupling to the plasma for an $n=5$ TAE similar to that achieved with the much bigger saddle coils for an $n=2$ TAE. Figure1 shows one of these two assemblies as installed in JET. The new diagnostic system has operational capabilities very similar to those of the saddle coils (frequency range $10 \mathrm{kHz} \leftrightarrow 500 \mathrm{kHz}$, maximum antenna current and voltage $\max \left(\mathrm{I}_{\mathrm{ANT}}\right) \sim 15 \mathrm{~A}$ and $\max \left(\mathrm{V}_{\mathrm{ANT}}\right) \sim 1 \mathrm{kV}$, respectively, and power $\left.\sim 5 \mathrm{~kW}\right)$ but now focuses specifically on the medium to high range of toroidal mode numbers $|n|=3 \div 20$ [5].

The typical values of the antenna current and voltage used for the AE excitation experiments are $\mathrm{I}_{\mathrm{ANT}} \sim 10 \mathrm{~A}$ and $\mathrm{V}_{\mathrm{ANT}} \sim 500 \mathrm{~V}$. As shown in fig2(a,b), this currents and voltages produce a very small vacuum radial magnetic field at the antenna position, $\left|\mathrm{B}_{\mathrm{r}}\right| \sim 0.5 \mathrm{G}$ for $\mathrm{I}_{\mathrm{ANT}}=1 \mathrm{~A}$, in turn driving a typical $\left|\mathrm{B}_{\mathrm{r}}\right| \sim 0.1 \mathrm{G}$ at the plasma edge and $\left|\mathrm{B}_{\mathrm{r}}\right| \sim 0.01 \mathrm{G}$ in the plasma core at the typical value of the total antenna current $\left(\mathrm{I}_{\mathrm{ANT}} \sim 10 \mathrm{~A}\right)$. Given the position of the new antennas in the poloidal crosssection of JET (which is sketched in fig7c), the lowest value of the edge elongation ( $\left.\kappa_{95}\right)$ at which a stable plasma configuration can be routinely produced so as to give a reliable density control and a sufficiently good antenna-plasma coupling (i.e. a large enough plasma minor radius a $>0.8 \mathrm{~m}$ with a distance between the last closed flux surface and the antennas $\mathrm{d}<100 \mathrm{~mm}$ ), is around $\kappa_{95} \sim 1.30$. Due to JET operational constraints, more circular plasmas with lower edge elongation $\kappa_{95}<1.25$ have much smaller minor radius $(\mathrm{a} \sim 0.7 \mathrm{~m})$ and are localised too far away from the $\mathrm{AE}$ antennas $(d>120 \mathrm{~mm})$, hence these situations are not routinely covered in our experimental studies.

The plasma response to the driven perturbation is measured via synchronous detection. A $1 \mathrm{kHz}-$ clock digital control system, the Alfvén Eigenmode Local Manager (AELM), is used to control the 
paper post IAEA-TCM-EP-2009: accepted for Nuclear Fusion Special Issue EP2010, March 2010

AE excitation in real time. The AELM sweeps linearly the antenna frequency around an initial guess for the AE resonance. The AELM is also used to detect and track in real-time the individual resonances corresponding to the antenna-driven, stable plasma modes. A fit of the complex antenna/plasma transfer function is then applied, to obtain the mode frequency and damping rate, as well as the mode amplitude at the different probe locations, with a typical time resolution of the order of $10 \div 20 \mathrm{~ms}$. Any combination of the 8 antennas can be chosen with different relative phasing ( + and - ) to excite different $n$-spectra, with measurable radial $B_{r}$ field up to $|n| \sim 20$, as shown in fig2(a-d). A very broad toroidal spectrum is excited for any antenna frequency, comprising many components, of which the higher-n ones are more strongly attenuated as function of the distance from the antennas, as shown in fig2c. By appropriate selection of the active antennas and their relative phasing, one can however drive predominantly n-odd or predominantly n-even excitation spectra, as well as producing larger components for lower-n $(|\mathrm{n}|<10)$ or higher-n modes $(8<|\mathrm{n}|<20)$, as shown in fig2d for two different JET discharges using the actual antenna current.

The damping rate of $|\mathrm{n}| \leq 2$ modes in ohmic limiter plasmas was found to be essentially identical to that measured with the saddle coil system $[13,14]$, confirming the robustness of the measurements made with the new antenna system. Despite the much lower radial magnetic field driven in the plasma by the new antennas (on the magnetic axis at $[R, Z]=[3,0.3] \mathrm{m}$ we have $\left|B_{\mathrm{r}}\right| \sim(5 \div 10) \times 10^{-4} \mathrm{G}$ for the $|n|=5 \div 10$ TAEs with the new antennas at the typical operating values of the antenna current $\mathrm{I}_{\mathrm{ANT}} \sim 10 \mathrm{~A}$ compared to $\left|\mathrm{B}_{\mathrm{r}}\right| \sim 5 \times 10^{-2} \mathrm{G}$ for the $|\mathrm{n}|=1$ and $|\mathrm{n}|=2$ TAEs driven by the saddle coils with current $\mathrm{I}_{\mathrm{SC}} \sim 5 \mathrm{~A}$ ), a new result was immediately observed, namely that many marginally stable harmonics with $|\mathrm{n}| \sim 3 \div 12$ and $\gamma / \omega<0.5 \%$ were found to be simultaneously excited at very close-by frequencies in the plasma, i.e. a frequency-degenerated spectrum where the half-width at halfmaximum of the modes (closely related to the damping rate) is comparable to their separation in frequency. This feature is clearly related to the very broadband spectrum driven by the new antenna system, and it has been the main motivation towards developing the use of a more sophisticated real-time and post-pulse algorithm for mode detection and tracking and n-number discrimination using the Sparse Signal Representation method and the SparSpec code [15], as other algorithms, for instance those based on the Single Value Decomposition (SVD) method, have been found to be inadequate to meet both the real-time and post-pulse measurement requirements. The SparSpec algorithm has been adapted from its original implementation for astronomy and astrophysics applications, which uses only real-valued data in a "post-measurements" analysis mode, to deal with complex (and real-time) data in fusion plasmas. This algorithm has been successfully validated and benchmarked on real and simulated JET data [16]. A clear illustrative example of the findings

\footnotetext{
- freeware available at: http://www.ast.obs-mip.fr/Softwares
} 
paper post IAEA-TCM-EP-2009: accepted for Nuclear Fusion Special Issue EP2010, March 2010

on this degenerate mode spectrum and on the data analysis method used to discriminate between its various components is shown in fig3(a,b) for a case of a JET ohmic plasma in limiter configuration. While in the spectrogram (fig3a) we can only identify three distinct bands where modes are actively driven and damping rate can be measured, indicated by the much smaller width of the sweep of the (triangular) antenna frequency waveform, it is only with the SparSpec code that we can ascertain that three separate $n=5, n=7$ and $n=11$ modes co-exist in the lowest frequency band around $160 \mathrm{kHz}$ (fig3b). Regarding fig3a, it is important to note that the resolution of the data acquisition system is such that modes with amplitude $|\delta \mathrm{B}| \sim 3 \times 10^{-4} \mathrm{G}$ at the plasma edge are already "barely" visible in the spectrogram for ohmic limiter plasmas, as for the three bands visible around $220 \mathrm{kHz}, 200 \mathrm{kHz}$ and $160 \mathrm{kHz}$, for which the edge amplitude is estimated to be around $|\delta \mathrm{B}| \sim 5 \times 10^{-4} \mathrm{G}$ for those time points where there is no direct drive from the active antennas. Conversely, it is not possible to know apriori if these very weak bands are in fact associated to stable modes $(\gamma / \omega>0)$ unless the antenna frequency waveform scans across them. Only in this case, in fact, the synchronous analysis of the antenna-driven phase relation between the [I, Q] (in-phase and quadrature) components of the measured fluctuation signals can provide such a discrimination [11]. Only for antenna-driven stable modes these two components describe a circle in the complex plane (see fig4 and fig5 for some specific examples) when plotted against each other, as this quadrature relation is imposed by the driving system itself, and this allows to separate conclusively the antenna from any plasma drive, which does not have a fixed and imposed [I, Q] phase relation.

Considering now the lower frequency band around $160 \mathrm{kHz}$ for those time points where the antenna frequency runs across it (i.e. when we can ascertain that the modes are stable, with $\gamma / \omega>0$ ), had we analysed it using a standard linear phase fitting algorithm to determine the mode numbers, we would have found an $n=8$ mode with a damping rate $\gamma / \omega \sim 2 \%$. Using SparSpec, we obtain $\gamma / \omega \sim 1.7 \%$ for the $n=5, \gamma / \omega \sim 0.9 \%$ for the $n=7$ and $\gamma / \omega \sim 0.45 \%$ for the $n=11$ modes, respectively, using a CPU time for this calculation of around $2 \mathrm{~ms}$ for each individual up/down frequency scan. Had we used an SVD decomposition algorithm similar to the one presented in [17], requiring a combinatorial exploration of all possible solutions and an a-posteriori thresholding scheme to determine the correct ones, we would have found very similar results as far as $\mathrm{n}$ and $\gamma / \omega$, but now using a CPU time usually in excess of $150 \mathrm{~ms}$ for each individual up/down frequency scan. The incredible speed and accuracy of the SparSpec algorithm has made it possible to deploy it in our real-time plant control software, which allows real-time detection and tracking of individual mode numbers within a CPU-time of $<600 \mu$ s for each $1 \mathrm{~ms}$ real-time clock cycle. This now allows a detailed quantitative analysis of these measurements, as mode numbers can be directly separated and reliably in real-time and individually tracked to measure the changes in the mode frequency and damping rate during the evolution of the plasma background. 
paper post IAEA-TCM-EP-2009: accepted for Nuclear Fusion Special Issue EP2010, March 2010

Regarding error analysis, the digital synchronous detection system allows for a very precise determination of the mode's frequency, as the bandwidth of the optical phase-lock loop used in the hardware is such that the frequency is known to within less than $50 \mathrm{~Hz}$. The accuracy of the absolute amplitude, mode number and damping rate of the synchronously detected magnetic perturbations is affected by the presence of background, un-coherent turbulence at the same frequency of the synchronous measurements, by electrical (white) noise in the measurements, and particularly any DC offset, which overall can lead to a typical synchronously detected signal-to-noise ratio $(\mathrm{S} / \mathrm{N})$ in the range $3<\mathrm{S} / \mathrm{N}<200$ (with values $\mathrm{S} / \mathrm{N}>100$ for the experiments reported here). Additionally, errors on the calibration of the complex raw signal from the different pick-up (Mirnov-type) coils and engineering signals (antenna current and voltages) used for the real-time and post-pulse analysis affects the accuracy of the amplitude, mode number and damping rate measurements. We estimate that the absolute value for the plasma response $\left|\delta \mathrm{B}_{\mathrm{r}}(\omega)\right|$ to the antenna-driven radial magnetic field $\left|\mathrm{B}_{\mathrm{r}}\right|$ is correct within a factor 2 for all frequencies, and as such it should be noted that this somewhat large uncertainty has only a minimal effect on the accuracy of the damping rate data, which are extracted from fitting $\delta \mathrm{B}_{\mathrm{r}}(\omega)$ in the complex plane. For the accuracy on the determination of the mode numbers one has to consider as well the possible statistical and systematic errors due to the numerical algorithm used to extract such data. Given the constraints imposed by SparSpec, the spectral window of the magnetic sensors used for this analysis, and the extensive tests carried out on real and simulated JET data, we have ascertained that the toroidal mode numbers can be determined exactly (i.e. $n=n \pm 0$ ) up to $|n| \sim 10-12$ for modes whose (normalized) amplitude is at least $35 \%$ of the maximum amplitude in the spectrum (depending on the amount of noise in the measurements, but provided the overall $\mathrm{S} / \mathrm{N}>10$ at least), with an error that can be up to \pm 2 for higher mode numbers and/or modes with lower (relative) amplitudes and/or for cases with a lower overall $\mathrm{S} / \mathrm{N}<5$. Finally, for the accuracy of the damping rate, one has to add the errors from the algorithm used to fit the measurements, leading in total to an uncertainty on $\gamma / \omega$ estimated to be around $30 \%$ for the typical cases that we consider in our analysis.

\section{The Dependence of the Damping Rate of $n=3$ and $n=7$ TAEs on the Edge Elongation}

As several different damping mechanisms have been theoretically proposed for AEs, systematic experimental studies are needed to obtain the dependence of their damping rate on the background plasma parameters. With this approach, one can then find the plasma parameters that are more important for the AE stability, hence determine and quantify with direct comparisons with model calculations the dominant damping mechanisms in current devices. From then on, not only will be it relatively simple to extrapolate with confidence to future devices such as ITER when considering 
paper post IAEA-TCM-EP-2009: accepted for Nuclear Fusion Special Issue EP2010, March 2010

the same experimental conditions, but one will also be able to devise those particular experimental conditions where usually less important damping mechanisms may become dominant, which is in fact what is theoretically foreseen to occur for ITER. For example, the ion and electron Landau damping and the radiative damping mechanisms contribute very little to the damping rate of low-n AEs in JET ohmic plasmas $[18,19]$, but are expected to be the dominant damping mechanisms in ITER for AEs with $\mathrm{n} \sim 5-10$ due to the much higher plasma temperature.

In JET, the edge plasma shape and magnetic shear have been found experimentally $[13,20]$ and theoretically $[21,22]$ to be key ingredients for increasing the damping of both stable, antennadriven low- $n(n=1, n=2)$ and unstable, fast-ion driven medium- $n(n \sim 3 \div 10)$ AEs. This has motivated previous experimental studies on the Alcator C-mod tokamak where, contrary to the JET results for $n=1$ and $n=2$ TAEs, it was found that the damping rate of an $n=6$ TAE remains essentially invariant when the average edge triangularity $\left(\delta_{95}\right)$ is scanned in the range $0.3<\delta_{95}<0.7$, which is also operationally associated to a similar variation in the edge elongation [23].

With the new set of JET antennas, and the real-time mode number discrimination algorithm recently implemented, it has now become possible to repeat the previous low-n measurements for modes with $|n| \sim 3 \div 12$. In this respect, the capability of a real-time detection and tracking of the individual $\mathrm{n}$-components in the antenna driven spectrum constitutes an invaluable tool, which is unique to JET and is lacking in other devices, to provide an accurate testing for the code predictions, as it is paramount that the same mode be measured throughout the parameter scan.

We show here the measurement of the damping rate for an $n=3$ (JET shot \#77788, fig4) and an $n=7$ (JET shot \#77790, fig5) TAE as function of the edge elongation $\left(\kappa_{95}\right)$ for ohmic plasmas. Neutral Beam blips 200ms-long were used for diagnostic purposes to measure the safety factor, the ion temperature and the toroidal rotation frequency profiles. These two discharges have very similar background plasma parameters (toroidal magnetic field $\mathrm{B}_{\phi}=2.7 \mathrm{~T}$, plasma current $\mathrm{I}_{\mathrm{p}}=2 \mathrm{MA}$, central and volume-averaged electron density $\mathrm{n}_{\mathrm{e} 0} \sim 2 \times 10^{19} \mathrm{~m}^{-3}$ and $<\mathrm{n}_{\mathrm{e}}>\sim 1 \times 10^{19} \mathrm{~m}^{-3}$, central electron and ion

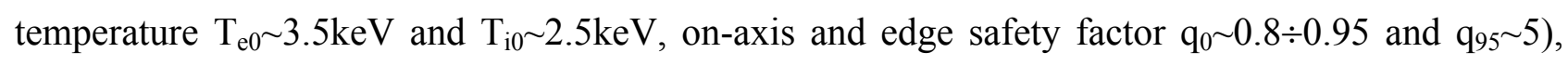
and are an exact match for the magnetic configuration and the plasma shape. Note also that at the higher end of the elongation scan, for $\mathrm{k}_{95}>1.5$, a lower $\mathrm{X}$-point is formed just above the divertor, but still outside the plasma cross section. Together with the main plasma parameters shown in fig4a and fig5a, in fig4(b-d) and fig5(b-d) we show some examples of the $n=3$ and $n=7$ resonances detected in the synchronously measured $\delta \mathrm{B}_{\mathrm{r}}$ spectrum and their fit in the complex plane. The very good quality of the fit is a clear indication of the accuracy of the damping rate measurements for each individual mode number.

The damping rate measurements for the $n=3$ and $n=7$ TAEs obtained during these two discharges are shown in fig6 together with the data calculated using the LEMan code, that will be discussed in 
paper post IAEA-TCM-EP-2009: accepted for Nuclear Fusion Special Issue EP2010, March 2010

Section-4. Considering now only the measurements of $\gamma / \omega$, we note an almost linear increase in the damping rate as a function of the edge elongation for these two modes. Hence, increasing the edge elongation has the same effect on the damping rate of these medium- $n$ TAEs as on the $n=1$ and the $\mathrm{n}=2$ TAEs, which is in clear contradiction with the measurements made for an $n=6$ mode in Alcator $\mathrm{C}$-mod. For otherwise very similar background plasma parameters, the damping rate for the $\mathrm{n}=3$ TAE increases from $\gamma / \omega \sim 0.3 \%$ at $\kappa_{95} \sim 1.33$, to $\gamma / \omega \sim 2.2 \%$ at $\kappa_{95} \sim 1.35, \gamma / \omega \sim 3 \%$ at $\kappa_{95} \sim 1.43$ and $\gamma / \omega \sim 5.5 \%$ for $\kappa_{95}>1.55$, hence overall a factor $\sim 20$ increase for a variation in the edge elongation of $\sim 0.2$. For the $n=7 \mathrm{TAE}$, this increase in the damping rate is even sharper, as $\gamma / \omega \sim 3 \%$ for $\kappa_{95} \sim 1.35$ and $\gamma / \omega \sim 6.5 \%$ for $\kappa_{95} \sim 1.43$, i.e. a factor $\sim 2.2$ increase over a variation of just $\sim 0.08$ in the edge elongation, which is very easily achieved in JET with only a minor increase (by about $\sim 5 \%$ ) in the current applied to the shaping coils. This result is very important because it confirms that the same damping mechanism acting upon global, low-n modes, plays a substantial role also for the stability of more core-localised medium-n TAEs, opening interesting perspectives for their real-time control. It is also to be noted that a sudden increase in the damping rate of the $n=3$ TAE occurs around $\kappa_{95} \sim 1.35$ : we see in fact that $\gamma / \omega<1 \%$ (and even as low as $\gamma / \omega \sim 0.5 \%$ ) for $\kappa_{95}<1.35$, whereas $\gamma / \omega>2 \%$ for $\kappa_{95}>1.35$. This is qualitatively similar to the observations made with respect to the earlier measurements for the $n=1$ and $n=2$ TAE modes [13], where a similar sudden jump in the values of $\gamma / \omega$ was observed around $\kappa_{95} \sim 1.5$. A somewhat similar feature also appears for the $n=7 \mathrm{TAE}$, but less convincingly as the data for $\kappa_{95}<1.35$ have been collected on other JET discharges which are not a sufficiently close match (i.e. within the error bar of the measurements) to the magnetic configuration and background plasma profiles (particularly the density) of \#77788 and \#77790. Although lacking for the time being a direct comparison of these more recent measurements with model calculations, we conjecture that this particular feature we observe for the scaling of the $n=3$ mode is due to an increased mode conversion to Kinetic Alfvén Waves (KAWs) at the plasma edge [22], i.e. a similar effect to that postulated to explain the dependence of the damping rate of the $n=1$ and $n=2$ modes on the plasma shape. Finally, note that as JET configurations with values of the edge elongation $\kappa_{95}<1.3$ cannot be routinely produced to give both a good antenna-plasma coupling and a reliable density control, it is not currently possible to determine whether there is a lowerbound in the edge elongation to determine the stability threshold $(\gamma / \omega=0)$ for these medium-n TAEs in ohmic plasmas, as the trend from higher towards lower values of $\kappa_{95}$ may appear to suggest..

\section{Model Calculation of the damping rate of $n=3$ TAEs using the LEMan code}

A model calculation of the damping rate of $n=3$ TAEs has been performed using the LEMan code $[7,8]$. LEMan is a full-wave code that has been designed to compute the propagation of waves in 
paper post IAEA-TCM-EP-2009: accepted for Nuclear Fusion Special Issue EP2010, March 2010

the Alfvén and Ion Cyclotron frequency ranges using a warm-plasma formulation for the dielectric tensor. This calculation of the dielectric tensor is based on the linearization of the Vlasov equation and on the Finite Larmor Radius (FLR) expansion where only terms of the order "0" are retained. This is sufficient to model the Kinetic Alfvén Wave and Landau damping and thus to evaluate the absorption of global modes like TAEs. Special attention has been given to the exact evaluation of the parallel wave vector. The latter has a strong impact on the wave propagation and the method required to retain its exact expression leads to a large increase of the computational resources required for these calculations.

LEMan does not include finite- $\beta$ effects because this would have required retaining terms up to second order in the FLR expansion. However, as in the ohmic discharges analysed here $\beta$ is very small $(\beta \sim 0.33 \%)$ and, following the work of Chu [24], we have empirically verified that for similar low- $\beta$ JET ohmic cases (and higher- $\beta$ case for TAEs driven by MeV energy ions in plasmas with a monotonic q-profile) one finds that $\mathrm{f}_{\mathrm{TAE}}(\beta=0) / \mathrm{f}_{\mathrm{TAE}}($ finite $-\beta) \sim 1+\beta(1+1 / \varepsilon)$, where $\varepsilon=\mathrm{a} / \mathrm{R}_{\mathrm{MAG}}$ is the plasma aspect ratio, finite- $\beta$ corrections are not deemed to be important in determining $\mathrm{f}_{\mathrm{TAE}}$ in low$\beta$ discharges as those analyzed here.

As LEMan deals with three-dimensional geometries, the equilibrium is first computed with the VMEC code [25], then mapped onto Boozer coordinates with TERPSICHORE [26]. This choice has the advantage of providing a sufficiently simple expression for the magnetic field while reducing the range of poloidal Fourier modes coupled to the antenna in the Alfvén domain of frequencies. The TERPSICHORE code has however not yet been adapted to geometries with an updown asymmetry. This implies that experimental measurements containing such asymmetry, which is inevitable in the presence (or when moving closer to the formation) of an X-point, cannot be handled exactly with the LEMan code, even in the plasma core.

From a numerical point of view, LEMan solves the Maxwell equations in term of potentials. This allows avoiding the so-called numerical pollution that can lead to unphysical results. A weak Galerkin form has been employed to solve the set of equations. The discretization is made in terms of Hermite cubic finite elements in the radial direction, as well as Fourier decomposition along the toroidal and poloidal angles. The radial meshing is sufficiently dense so as to model spiky shapes in the Eigenfunction. Furthermore, as a test of our calculations, we doubled the number of points in the radial meshing and the same plasma response was found, which convincingly proves that the resolution is sufficient at least to model the peaks seen in the scans we have performed.

For the simulations presented in this Section, all poloidal mode numbers $(\mathrm{m})$ from $\mathrm{m}=-5$ to $\mathrm{m}=22$ have been retained. As it is hard to know which ones of them are driven in the experiment (the antennas are all located at one single position in the poloidal plane), the elements of the poloidal Fourier series that models the localisation of the antenna are assumed to be of the same value. For 
paper post IAEA-TCM-EP-2009: accepted for Nuclear Fusion Special Issue EP2010, March 2010

the last case presented here a separate simulation was run including all poloidal components up to $\mathrm{m}=25$ as even the $\mathrm{m}=20$ harmonic has a non-negligible amplitude at the plasma edge: the results for these two cases are very similar, as shown later.

The comparison with the experimental measurements is performed for the $n=3$ TAE in the JET discharge $\# 77788$ at the following three time points: $t=6.113 \mathrm{sec}, \mathrm{t}=10.096 \mathrm{sec}$ and $\mathrm{t}=14.109 \mathrm{sec}$. The equilibrium is reconstructed using the data fitted by the EFIT code [27]. It has to be mentioned that in the LEMan code, the radial coordinate $s(s=0$ in the centre and $s=1$ at the border of the plasma) is defined as the toroidal flux instead of the square root of the poloidal flux as in most of the usual 2D codes. Hence, all data have been converted to this radial label, including those employed in the VMEC and TERPSICHORE codes; the simulation results are displayed using this representation. The results of these calculations, together with the profiles of the main background plasma parameters and the magnetic configuration, are shown in fig7(a-c) for the data at $\mathrm{t}=6.113 \mathrm{sec}$, fig8(ac) for the data at $\mathrm{t}=10.096 \mathrm{sec}$ and fig $9(\mathrm{a}-\mathrm{c})$ for the data at $\mathrm{t}=14.109 \mathrm{sec}$, respectively. Each figure is presented in three separate subplots: in subplot-a, we show the antenna-plasma loading, the different branches of the continuum spectrum around the frequency of the TAE gap, the Eigenfuction for different poloidal components for the parallel vector potential $A_{\|}$and the integrated power absorption; in subplot-b, we show the radial profiles of the electron density, electron and ion temperature, safety factor, magnetic shear, elongation and triangularity; in subplotc, we show the EFIT reconstruction of the poloidal cross-section of the plasma.

The gap formed by toroidicity is easily recognizable in the top frame of subplot-a: several peaks are visible in the antenna-plasma loading at different frequencies, and some of these peaks are located inside the TAE gap. When performing the experimental measurements, it is impossible to observe all of them, as the "real" antenna system excites and detects only one frequency at any one given time. It is straightforward to establish to which of those simulated peaks corresponds the measured TAEs, when considering the frequency range covered by the antenna sweep. For example, for the case at $\mathrm{t}=6.113 \mathrm{sec}$ ( shown in fig7), the experimental frequency scan is essentially located between $180 \mathrm{kHz}$ and $210 \mathrm{kHz}$. The TAE mode in LEMan is then to be found in this domain. The only peak satisfying this condition in the top frame of fig $7 \mathrm{a}$ has a frequency of $195 \mathrm{kHz}$, which agrees very well with the experimental data (measured mode frequency $\mathrm{f}_{\mathrm{MEAS}}=196 \mathrm{kHz}$ ). Once the frequency of the calculated mode has been matched to the experimental value, the damping is then simply evaluated by determining the width of the loading peak in the LEMan frequency scan, which gives $\gamma / \omega_{\mathrm{CALC}}=1.1 \%$, again in excellent agreement with the experimental value of $\gamma / \omega_{\mathrm{MEAS}}=1.2 \%$. In the middle frame of fig $7 \mathrm{a}$, the Eigenfunction of this $n=3$ TAE is represented: from $s=0.4$ outward the characteristic structure of a global mode is clearly recognizable, as it involves a large number of Fourier poloidal components from $\mathrm{m}=5$ to $\mathrm{m}=14$. In the region closer to the magnetic axis, the 
paper post IAEA-TCM-EP-2009: accepted for Nuclear Fusion Special Issue EP2010, March 2010

Alfvén branches $m=3$ to $m=5$ intersect with the continuum, which contributes considerably to the power absorption. This is obvious when looking in details at the bottom frame in fig7a, which represents the integrated power deposited from the centre to the label s under consideration. The steeper the trend, the more localized is the power absorption. For this particular case, most of the absorption occurs around $\mathrm{s} \sim 0.15$, i.e. it is dominated by the two $\mathrm{m}=3$ and $\mathrm{m}=4$ poloidal harmonics, and it is essentially due to radiative damping [28, 29] and mode conversion to Kinetic Alfvén Waves. Some absorption also occurs between $\mathrm{s} \sim 0.7$ and $\mathrm{s} \sim 0.85$, which is caused by electron Landau damping and, again, mode conversion to KAWs.

For the second situation at $\mathrm{t}=10.096 \mathrm{sec}$ ( code at $\mathrm{f}=171 \mathrm{kHz}$, which can be easily recognised in the plasma response scan displayed in the top frame of fig8a (the experimental frequency scan being located between $\sim 160 \mathrm{kHz}$ and $\sim 190 \mathrm{kHz}$, with $\mathrm{f}_{\text {MEAS }}=179 \mathrm{kHz}$ ). The calculated value of the frequency is $\sim 4.5 \%$ below the experimentally determined frequency at $t=10.073 \mathrm{sec}$. A simple explanation for this difference is related to the neutral beam blip injected from $t=10 \mathrm{sec}$ for diagnostics purpose. Between the time at which the $\mathrm{n}=3$ mode is measured and that at which the background plasma profiles are determined (used as input for the LEMan code), the density has slightly increased. This implies a lower value for the simulated TAE frequency and partly explains the difference between the experimental and the numerical results. Concerning the damping, a very good agreement has again been found between the measurement and the LEMan calculations, $\gamma / \omega_{\mathrm{CALC}} \sim \gamma / \omega_{\mathrm{MEAS}} \sim 2 \%$. As shown in the bottom and middle frame of fig8a, respectively, the power absorption and the Eigenfunction (computed using all poloidal harmonics between $\mathrm{m}=2$ and $\mathrm{m}=15$ ) are very similar to those shown in fig7a for the first time point, with an additional power absorption step around $s \sim 0.1$.

Finally, for the simulation at $\mathrm{t}=14.109 \mathrm{sec}$, a peak corresponding to an $\mathrm{n}=3$ TAE is identified at $\mathrm{f}=183 \mathrm{kHz}$ (as shown in the top frame of fig9a), again in good agreement with the experimental measurement of the mode frequency (the experimental frequency scan being located between $\sim 167 \mathrm{kHz}$ and $\sim 198 \mathrm{kHz}$, and $\mathrm{f}_{\mathrm{MEAS}}=180 \mathrm{kHz}$ ). Conversely, the calculated damping rate $\gamma / \omega_{\mathrm{CALC}}=2.0 \%$ is much smaller than the measured value, $\gamma / \omega_{\mathrm{MEAS}} \sim 4.2 \%$. To give an explanation for this clear discrepancy, fig11 (which will be discussed later) displays the original experimental equilibrium and the one used in the LEMan simulations by retaining the up-down symmetry. As shown in the middle frame of fig9a, the global mode is constituted of a larger number of poloidal harmonics than before (from $\mathrm{m}=2$ to $\mathrm{m}=20$ ). This is due the q-profile having changed significantly compared to the first two cases, particularly towards the plasma edge: $q$ now reaches $q(s=1) \sim 5.60$ at the last closed flux surface. The integrated power in the bottom frame of fig9a shows that the most of absorption is now located towards the plasma edge for $\mathrm{s}>0.7$, implying that the proportion of the absorption located in the TAE continuum (at the plasma edge) compared to the part related to mode 
paper post IAEA-TCM-EP-2009: accepted for Nuclear Fusion Special Issue EP2010, March 2010

conversion with the harmonics $m=3$ to $m=5$ (in the plasma core) is much more important now than at $\mathrm{t}=6.113 \mathrm{sec}$ and $\mathrm{t}=10.096 \mathrm{sec}$.

As a larger number of poloidal harmonics now make up the $n=3$ global mode, a second simulation for this time point was run using all Fourier components with $-5 \leq m \leq 25$, with the results shown in fig10. The frequency spectrum, Eigenfunction, power absorption and the resulting damping rate are substantially similar in these two runs, which excludes the role of different poloidal spectra as a possible cause for the discrepancy between the measured and computed damping rate. A likely cause for this disagreement can be found by looking in more details at the edge plasma shape used in the simulations, which is shown in fig 11 . We note that at $t=6.113 \mathrm{sec}$ (fig7, first case) and $\mathrm{t}=10.096 \mathrm{sec}$ (fig8, second case), the up-down symmetry is well satisfied and the magnetic configurations used in the simulation are very close to the experimental ones. This is no longer the case in the third simulation at $\mathrm{t}=14.109 \mathrm{sec}$ (fig9), where a lower X-point appears because of the much higher edge elongation. The simulation cannot then be performed on the "real" equilibrium (i.e. as given by EFIT): our ansatz is that it is this difference in mapping the exact shape of the JET poloidal cross-section that explains why the damping rate evaluated with the LEMan code is not very precise in this particular case.

Concerning the possible role of the electron density and q-profiles to explain the difference between measurements and LEMan calculations of the damping rate for these $n=3$ TAEs, the calculated $\gamma / \omega$ is indeed sensitive to those values, and this could provide another explanation for the divergence between the experimental and the simulation results. However, these parameters also act on the TAE frequency (for the electron density through the ion density in supposing quasi-neutrality). Thus a sufficiently large disagreement would also have to be found for this quantity, which is not the case. Further work is now underway in order to clarify convincingly which of those effects is the more relevant to explain the damping rate discrepancy. This work includes comparisons with other numerical codes, such as LIGKA [30], CASTOR [31], TAEFL [32] and GYGLES [33, 34], and using additional measurement points taken immediately before the formation of the up-down asymmetry in the plasma poloidal cross-section.

\section{Outlook and Future Work}

The first measurements of antenna-driven AEs with toroidal mode number in the range $|\mathrm{n}| \sim 3 \div 15$ have convincingly demonstrated that many of such modes exist at very similar frequencies in the plasma rest frame, such that their frequency separation is less than the modes' half-width at halfmaximum, i.e. the damping. This has prompted the development of a sophisticated real-time mode number detection and discrimination algorithm for such a frequency-degenerated spectrum, which is based on the sparse representation of signals. Routine measurements of the damping rate for low- 
paper post IAEA-TCM-EP-2009: accepted for Nuclear Fusion Special Issue EP2010, March 2010

and medium- to high-n AEs have now been obtained for various JET operating regimes, with realtime tracking of the driven resonances providing tens of damping rate data for each individual mode number on a single discharge. This result has been obtained using compact antennas with a small effective area, furthermore located rather far away from the plasma edge, at a typical distance in excess of $60 \mathrm{~mm}$ from the last closed flux surface. This is therefore a very promising technical result in view of a possible use of compact active antennas in ITER for burn control applications.

The first quantitative analysis of the damping rate measurements obtained for medium- $n$ TAEs, $n=3$ and $n=7$, has confirmed the experimental scaling of an increase in $\gamma / \omega$ as the edge elongation (hence the edge magnetic shear) is increased. This measured scaling is in agreement not only with previous measurements in JET for low-n TAEs, but also with previous theoretical estimates based on mode conversion of TAEs to Kinetic Alfvén Waves. However, this contradicts results obtained in the Alcator C-mod tokamak, and among the possible reasons for this discrepancy is the absence in this device of real-time tracking or post-pulse discrimination of the individual mode numbers making up a degenerate frequency spectrum, which in turns makes it uncertain that the same mode is followed throughout the dynamical evolution of the plasma and the particular parameter scan under study.

The absence of both real-time tracking and n-number discrimination of the antenna-driven modes makes it impossible to obtain a repeated sequence of resonances that are effectively guaranteed to be associated to the same mode, particularly when the driven antenna spectrum contains many frequency-degenerated components with similar amplitude. As an example, in Alcator C-mode experiments, only a couple of resonances are usually obtained on each discharge, for which the mode number cannot be discriminated in real-time, whereas on JET, depending on the selection of certain options in the real-time algorithm we use, we can routinely obtain $>50$ resonances for each individual n-number components in the driven antenna spectrum on each discharge. This allows for a conclusive and unambiguous measurement of the dependence of the damping rate for each individual mode numbers during a controlled scan of a given plasma parameter on the same discharge.

The first quantitative measurements of the damping rate for individual mode numbers made in JET have now been made available for detailed comparisons with theory and models. A first benchmark has been performed using the LEMan code for an $n=3$ TAE, which has conclusively demonstrated the importance of retaining the up/down asymmetry in the plasma poloidal cross section in order to be able to quantitatively reproduce the measured scaling of the damping rate for this mode as function of the edge elongation. For the cases where the plasma poloidal cross section is sufficiently up/down symmetric, the LEMan results for the mode frequency and damping rate are in very good agreement with the measurements, and also demonstrate that a large number of poloidal harmonics 
paper post IAEA-TCM-EP-2009: accepted for Nuclear Fusion Special Issue EP2010, March 2010

should be used so as to reproduce correctly the measured $\gamma / \omega$. Further benchmark work with other codes is the subject of ongoing work within the ITPA work-programme.

Together with the ability of detecting and correctly separating in real-time individual mode numbers in a multi-component stable AE spectrum, the confirmation, first reported in this work, of the low-n scaling of $\gamma / \omega=\mathrm{f}\left(\kappa_{95}\right)$ for the more interesting medium-n TAEs, as these are fast ion (and specifically $\alpha$ 's) driven modes, opens the door for the implementation of a closed real-time loop for the control of the TAE stability in burning plasmas, such as the regimes foreseen for ITER. The damping rate measurements are already available in the JET real-time signal server, and their real-time estimate has been shown to be in very good agreement with the result obtained with a more detailed postpulse analysis. To simulate in JET plasmas the destabilising contribution of the $\alpha$-particle drive, one could use the anisotropic MeV energy ions produced by Ion Cyclotron Frequency Heating or the mostly-isotropic, but lower energy, $\sim 150 \mathrm{keV}$ Neutral Beam ions. When the mode's damping rate reduces too much, the edge elongation can be increased via a small increase in the current (i.e. the actuator) of the shaping coils, so as to bring the plasma back to a situation where it is further away from the marginal stability limit.

On the more technical aspects, an upgrade in the excitation system is now planned for the near-term future, so as to be able to drive independently all the eight antennas and at higher power. This could create further burning plasma control situations, where the antennas are used to excite modes at higher amplitude to cause a controlled redistribution of the fast particles and prevent an excessive peaking of their pressure gradient. This would then reduce the risk of a more violent fast ion redistribution, which could lead to a total loss of ignition and plasma confinement.

\section{Acknowledgements}

This work, supported by the European Communities under the contract of Association between EURATOM and CRPP-EPFL, was carried out within the framework of the European Fusion Development Agreement. The views and opinions expressed herein do not necessarily reflect those of the European Commission.

The Authors would like to thank the various members of the CRPP, MIT and JET staff that have contributed to the design, installation, commissioning and routine operation of the new TAE antenna system. The Authors would particularly like to acknowledge the contribution of Alex Klein (formerly at MIT) and Alex Goodyear (CCFE) for their work on the development of the real-time application of the SparSpec code, and Simon Pinches (CCFE) for his assistance in producing the equilibrium data used as input for the LEMan code. 


\section{References}

1. W.W.Heidbrink, Phys. Plasmas 9(5) (2002), 2113.

2. G.Vlad, S.Briguglio, G.Fogaccia, F.Zonca, Nucl. Fusion 46 (2006), 1.

3. K.L.Wong, Plasma Phys. Control. Fusion 41 (1999), R1.

4. N.N.Gorelenkov, H.L.Berk, R.Budny, C.Z.Cheng, G.Y.Fu, W.W.Heidbrink, G.J.Kramer, D.Meade, R.Nazikian, Nucl. Fusion 43 (2003), 594.

5. D.Testa, A.Fasoli, et al., The new Alfvén Wave Active Excitation System at JET, Proceedings $23^{\text {rd }}$ Symposium on Fusion Technology (SOFT), Venice (Italy), 20-24 September 2004; weblink: http://infoscience.epfl.ch/record/143354/files/.

6. D.Testa T.Panis, P.Blanchard, H.Carfantan, A.Fasoli, Measurement of the Damping Rate of High-n Toroidal Alfvén Eigenmodes in JET, Proceedings $11^{\text {th }}$ IAEA Technical Committee Meeting on Energetic Particles, Kyiv (Ukraine), 21-23 September 2009; weblink: http://infoscience.epfl.ch/record/143355/files/.

7. P.Popovich, W.A.Cooper, L.Villard, Comput. Phys. Comm. 175 (2006), 250.

8. N.Mellet, Propagation and absorption of low frequency waves in two and three dimensional warm plasmas, CRPP-EPFL PhD Thesis (weblink: http://library.epfl.ch/theses/?nr=4398); submitted for publication to Computational Physics Communication, March 2010.

9. T.Panis, D.Testa, A.Fasoli, et al., Optimization of the Active MHD Spectroscopy System on JET for the Excitation of Individual Intermediate and High-n Alfvén Eigenmodes, Proceedings $11^{\text {th }}$ IAEA Technical Committee Meeting on Energetic Particles, Kyiv (Ukraine), 21-23 September 2009; weblink: http://infoscience.epfl.ch/record/142715/files/.

10. T.Panis, D.Testa, A.Fasoli, et al., Optimization of the Active MHD Spectroscopy System on JET for the Excitation of Individual Intermediate and High-n Alfvén Eigenmodes, accepted for publication in this Nuclear Fusion Special Issue.

11. A.Fasoli, D.Borba, G.Bosia, et al., Phys. Rev. Lett. 75 (1995), 645.

12. L.Villard, K.Appert, R.Gruber, J.Vaclavik, Comput. Phys. Reports 4 (1986), 95.

13. S.Bourguignon, H.Carfantan, T.Böhm, Astronomy and Astrophysics 462 (2007), 379.

14. D.Testa, A.Fasoli, Nucl. Fusion 41 (2001), 809.

15. A.Fasoli, C.Gormezano, H.L.Berk, et al., "Progress in the ITER Physics Basis Chapter 5: Physics of Energetic Ions”, Nucl. Fusion 47 (2007), S264.

16. A.Klein, H.Carfantan, D.Testa, A.Fasoli, J.A.Snipes, Plasma Phys. Control. Fusion 50 (2008), 125005.

17. M.Hole, L.Appel, Plasma Phys. Control. Fusion 49 (2007), 1971.

18. D.Testa, G.Y.Fu, A.Jaun, A.Fasoli, O.Sauter, Nucl. Fusion 43 (2003), 479.

19. D.Testa, A.Fasoli, A.Jaun, Nucl. Fusion 43 (2003), 724. 
paper post IAEA-TCM-EP-2009: accepted for Nuclear Fusion Special Issue EP2010, March 2010

20. D.Testa, A.Fasoli, D.Borba et al., Plasma Phys. Control. Fusion 46 (2004), S59.

21. A.Jaun, A.Fasoli, J.Vaclavik, L.Villard, Nucl. Fusion 40 (2000), 1343.

22. A.Fasoli, A.Jaun, D.Testa, Phys. Lett. A265 (2000), 288.

23. J.A.Snipes, N.Basse, C.Boswell, E.Edlund, A.Fasoli, Phys. Plasmas 12 (2005), 056102.

24. M.S.Chu, J.M.Greene, L.L.Lao, A.D.Turnbull, M.S.Chance, Phys. Fluids B4 (1992), 3713.

25. S.P.Hirshman, Phys. Fluids 26 (1983), 3553.

26. D.V.Anderson, W.A.Cooper, R.Gruber, S.Merazzi, U.Schwenn, J. Supercomp. Appl. (1990), 4, 34-47.

27. L.L.Lao, H.St.John, R.D.Stambaugh, A.G.Kellman, W.Pfeiffer. Nucl. Fusion 25 (1985), 1611.

28. C.Cheng, Phys. Rep. 211 (1992), 1.

29. G.Y.Fu, C.Z.Cheng, R.Budny, et al., Phys. Plasmas 3 (1996), 4036.

30. Ph.Lauber, S.Günter, A.Könies, S.D.Pinches, Journal Computational Physics 226 (2007), 447.

31. W.Kerner, J.P.Goedbloed, G.T.A.Huysmans, S.Poedts, E.Schwarz, Journal of Computational Physics 142 (1998), 271.

32. D.A.Spong, B.A.Carreras, C.L.Hedrick, Phys. Fluids B4 (1992) 3316.

33. R.Hatzky, M.Fivaz, Proceedings $25^{\text {th }}$ EPS Conference on Controlled Fusion and Plasma Physics, Prague (1998) (Petit-Lancy: European Physical Society, 1998), 1804.

34. M.Fivaz, S.Brunner, G.deRidder, et al., Comput. Phys. Commun. 111 (1998), 27. 


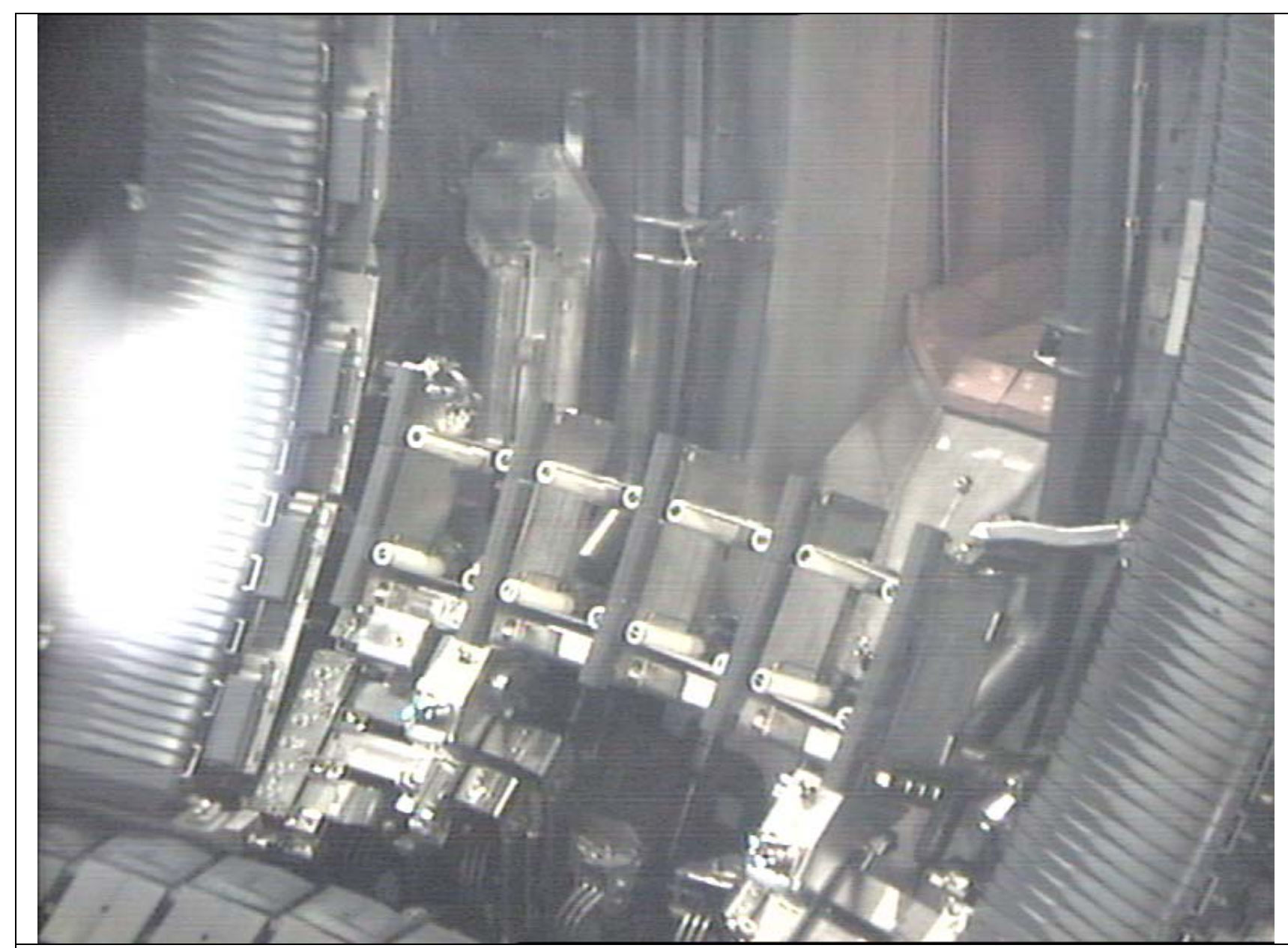

Figure1: view of one group of four AE antennas as installed in vessel during June 2005. D.Testa, Figure1, paper NF-2010 post IAEA-TCM-EP-2009-ACCEPTED 


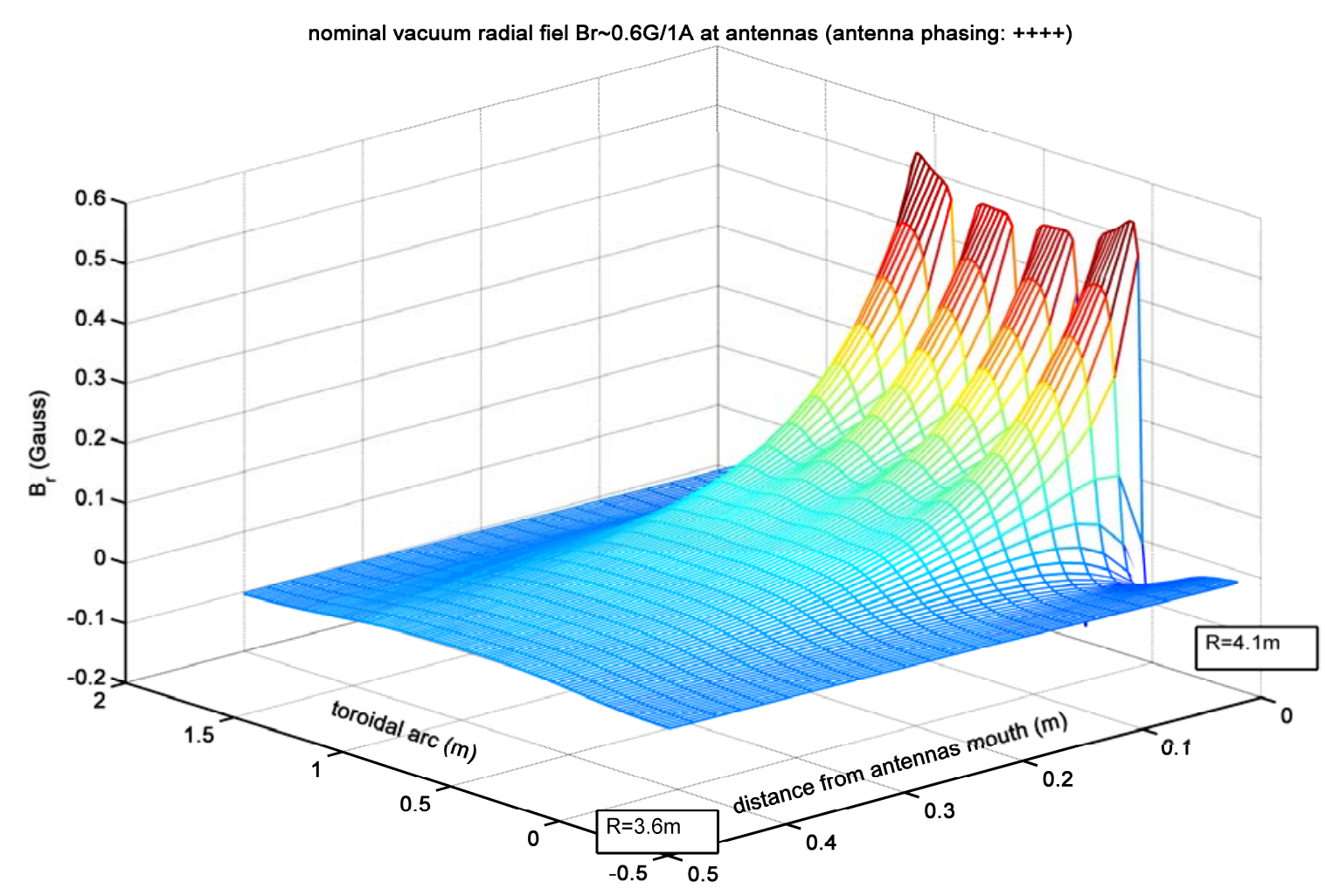

Figure2a: the total radial nominal magnetic field driven in vacuum by four neighbouring antennas in the same octant with the same ++++ phasing and a nominal antenna current $\mathrm{I}_{\mathrm{ANT}}=1 \mathrm{~A}$; the value $\mathrm{R}=4.1 \mathrm{~m}$ indicates the antenna position along the major radius coordinate, and $\mathrm{R}=2.98 \mathrm{~m}$ is the geometrical centre of the JET tokamak. Note the rapid exponential decay of the antenna-driven radial magnetic field as function of the distance from the antenna, so that $\max \left(\left|\mathrm{B}_{\mathrm{r}}\right|\right) \sim 0.01 \mathrm{G}$ in the plasma core even at the maximum antenna current allowable for operation $\left(\mathrm{I}_{\mathrm{ANT}} \sim 10 \mathrm{~A}\right)$.

D.Testa, Figure2a, paper NF-2010 post IAEA-TCM-EP-2009-ACCEPTED 


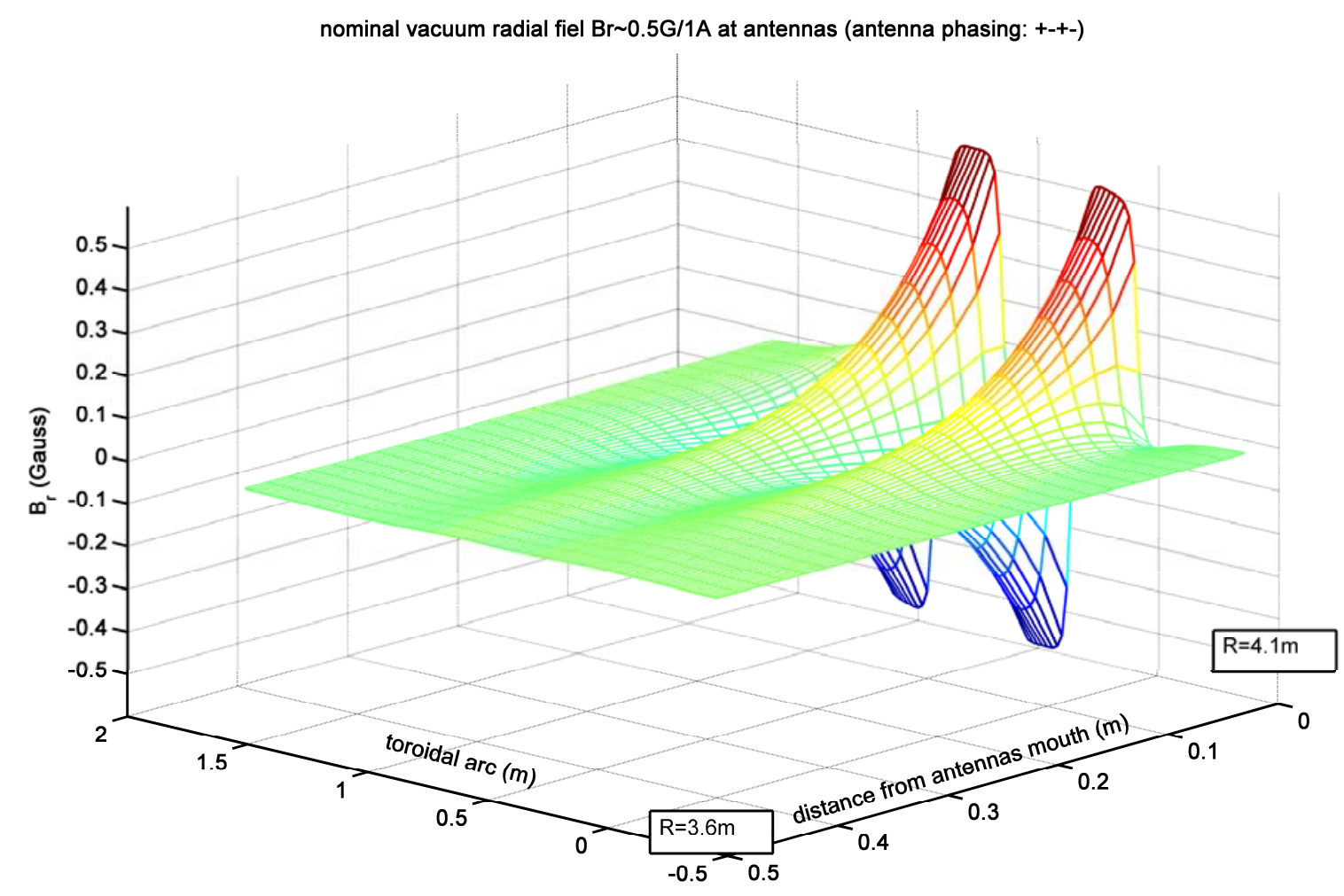

Figure2b: the total radial nominal magnetic field driven in vacuum by four neighbouring antennas in the same octant, now with opposite +-+- phasing and a nominal antenna current $\mathrm{I}_{\mathrm{ANT}}=1 \mathrm{~A}$. The exponential decay of the antenna-driven radial magnetic field is even faster for this antenna configuration than for the ++++ case, as in fact $\max \left(\left|\mathrm{B}_{\mathrm{r}}\right|\right) \sim 0.005 \mathrm{G}$ in the plasma core even at maximum antenna current.

D.Testa, Figure2b, paper NF-2010 post IAEA-TCM-EP-2009-ACCEPTED 


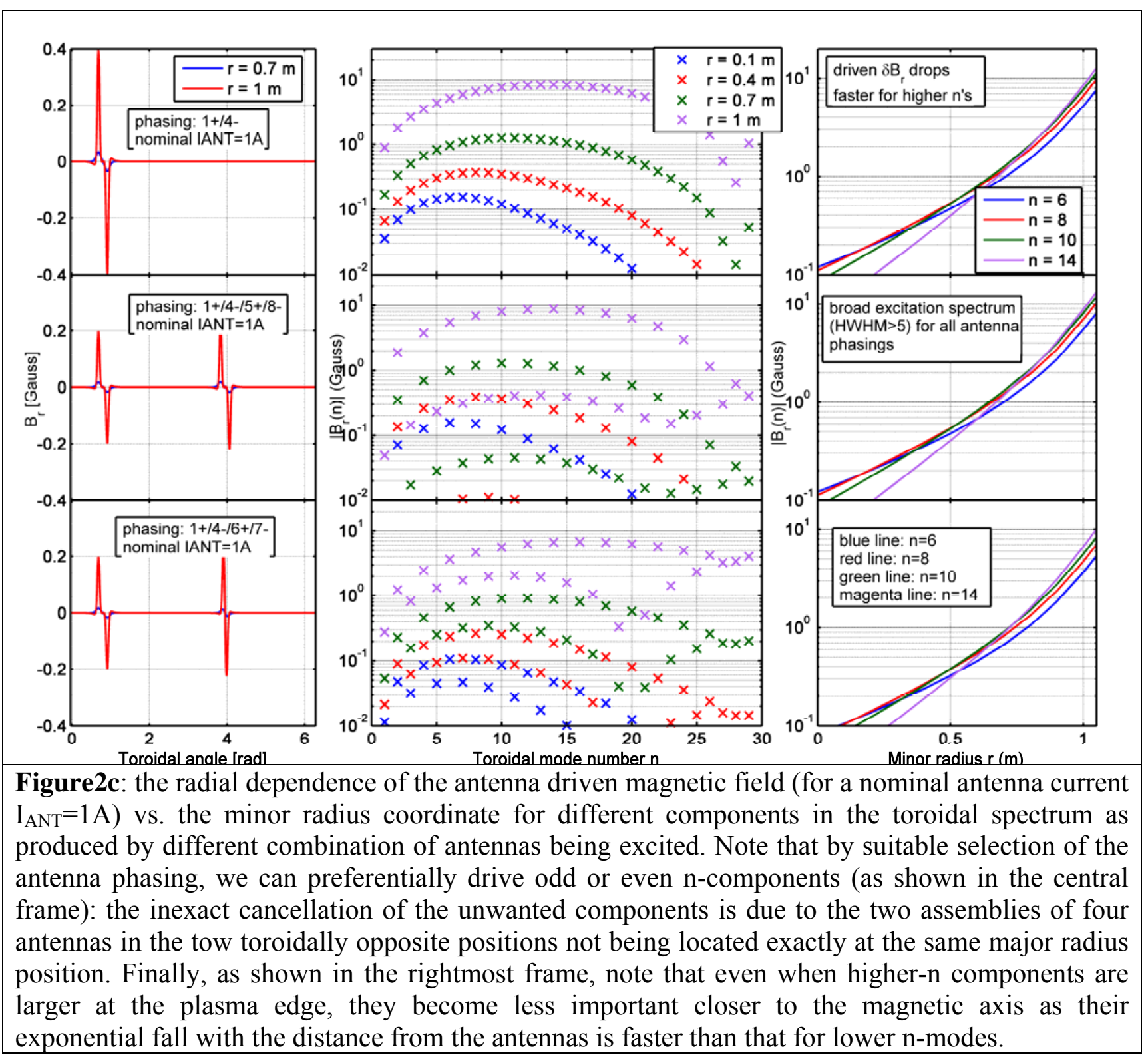

D.Testa, Figure2c, paper NF-2010 post IAEA-TCM-EP-2009-ACCEPTED 


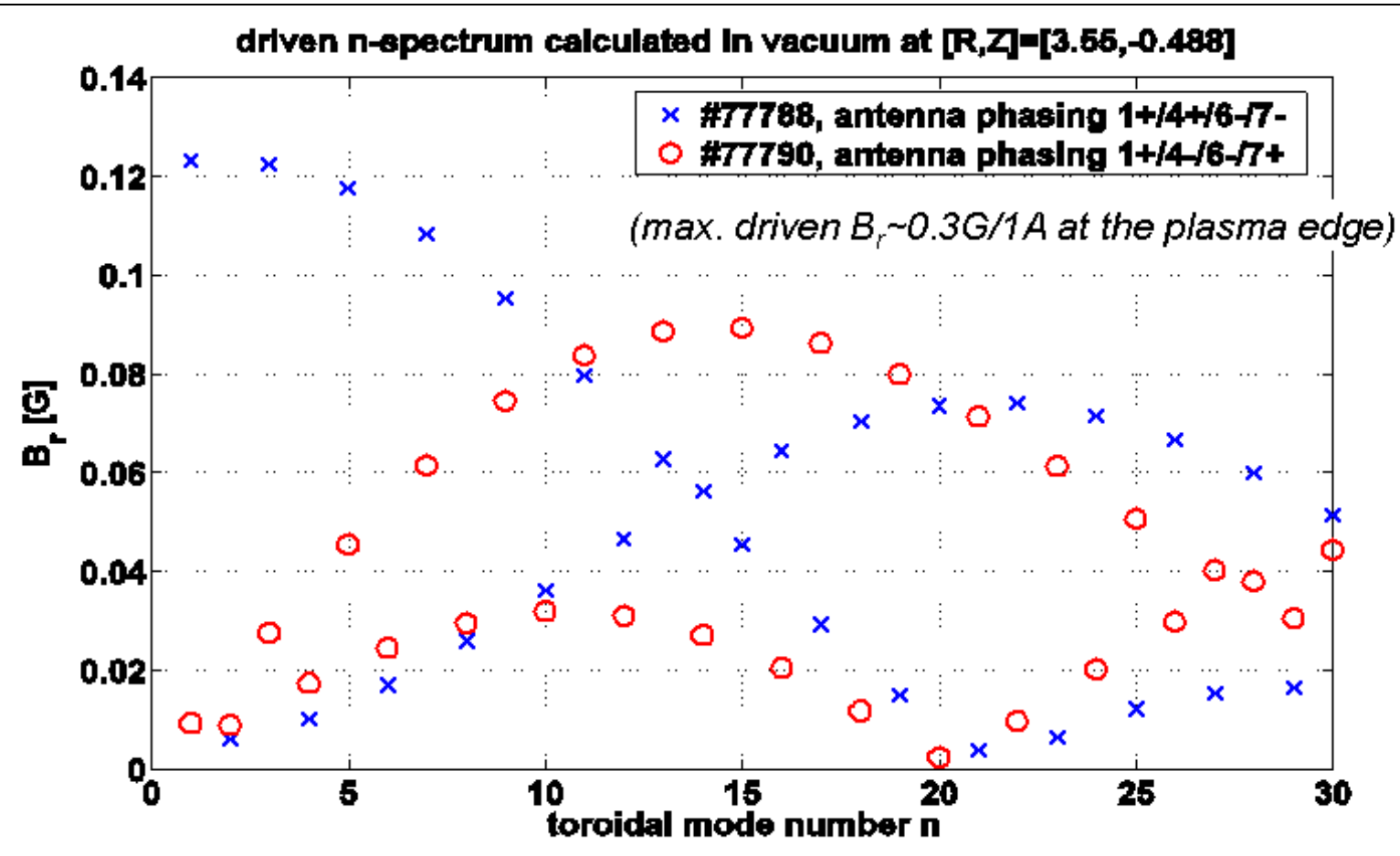

Figure2d: two examples of the toroidal spectrum of the antenna driven radial magnetic field, calculated using the actual antenna current $\mathrm{I}_{\mathrm{ANT}}$ driven in each discharge and the plasma geometry: using different antenna phasing, we can drive predominantly (in this odd) $n<5$ or $n \sim 6-15$ modes. Note again the non-exact cancellation of the unwanted ( $n=$ even) spectrum components.

D.Testa, Figure2d, paper NF-2010 post IAEA-TCM-EP-2009-ACCEPTED 


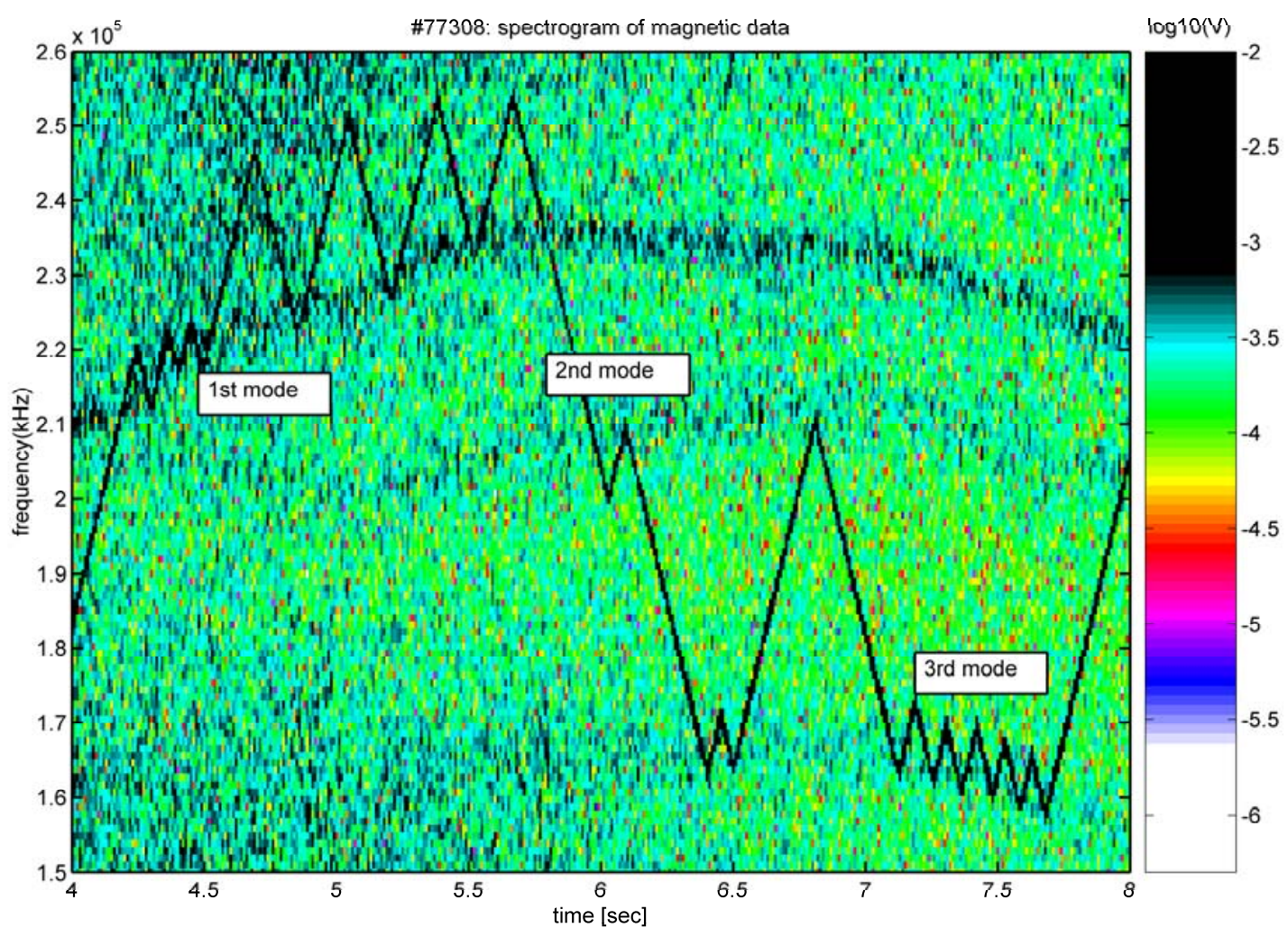

Figure3a: spectrogram of the magnetic perturbation measured for the JET shot \#77308: the antenna frequency scan is shown by the triangular sweeping waveform, and three different bands where plasma modes are excited by the AE antennas are observed; however, it is only with the SparSpec analysis shown in fig $3 \mathrm{~b}$ that we can determine precisely the mode numbers.

D.Testa, Figure3a, paper NF-2010 post IAEA-TCM-EP-2009-ACCEPTED 


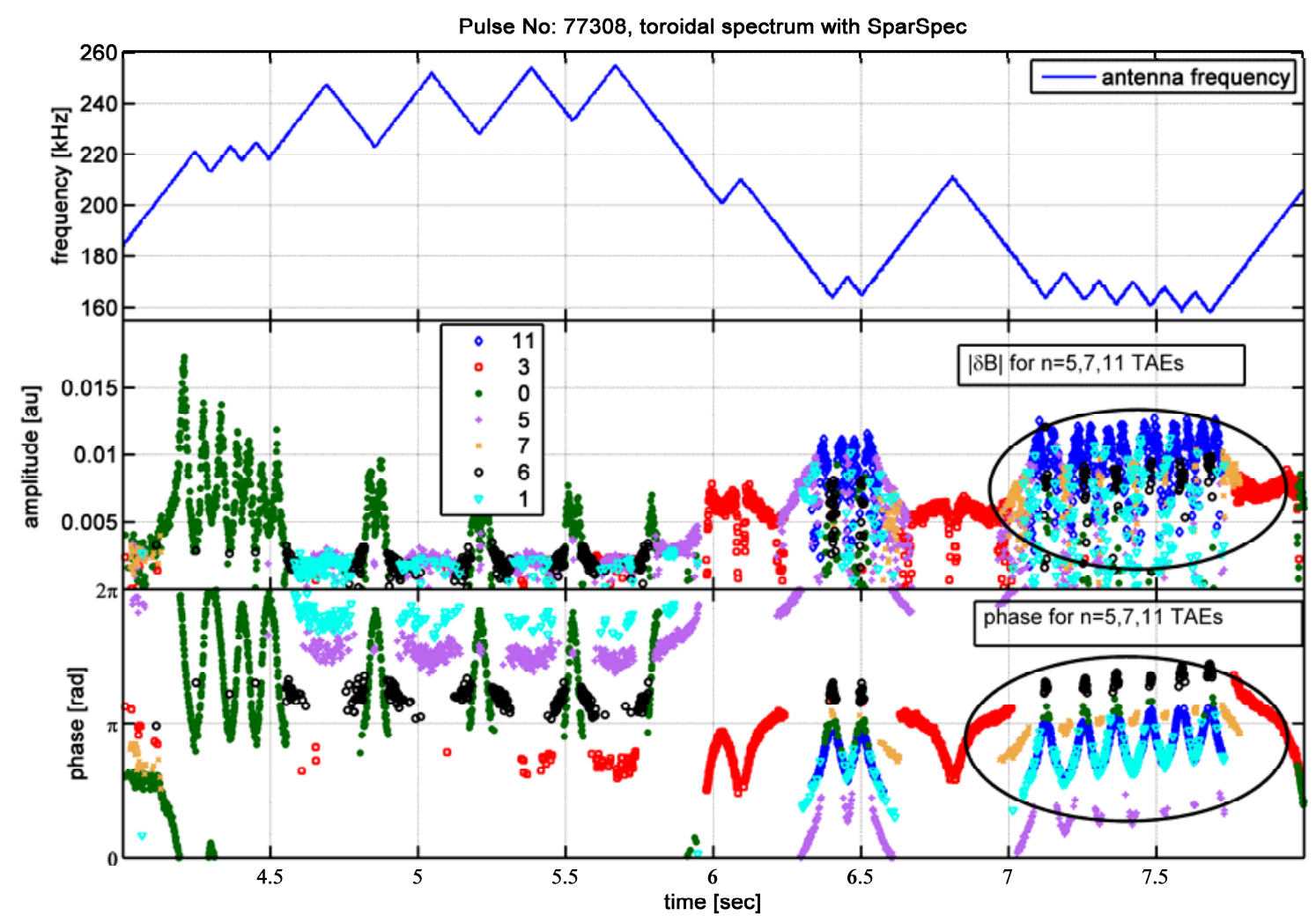

Figure3b: SparSpec post-pulse analysis of the synchronously detected data corresponding to the spectrogram shown in fig3a: we find that three separate $n=5, n=7$ and $n=11$ coexist in the lowest frequency band around $160 \mathrm{kHz}$.

D.Testa, Figure3b, paper NF-2010 post IAEA-TCM-EP-2009-ACCEPTED 


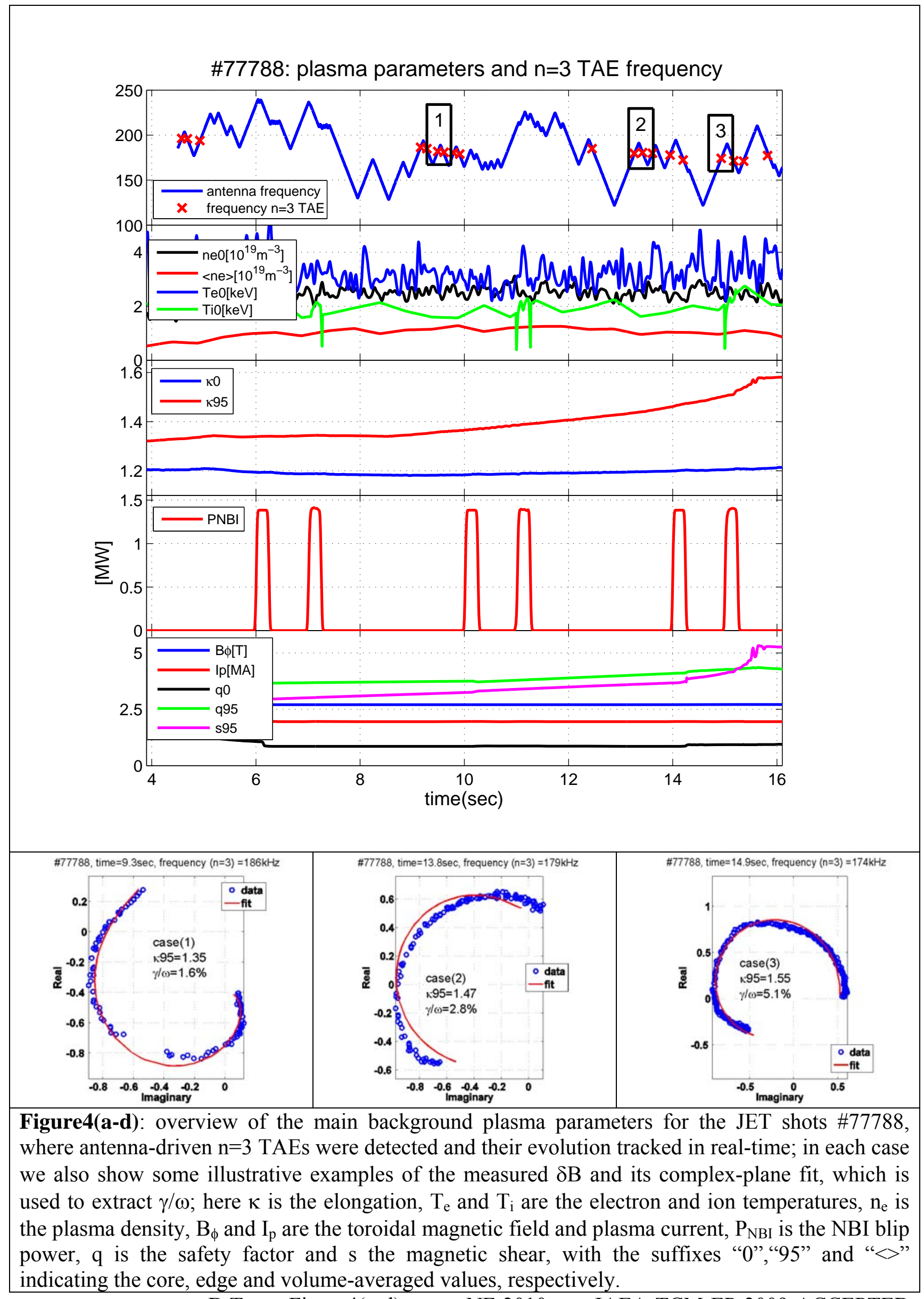

D.Testa, Figure4(a-d), paper NF-2010 post IAEA-TCM-EP-2009-ACCEPTED 


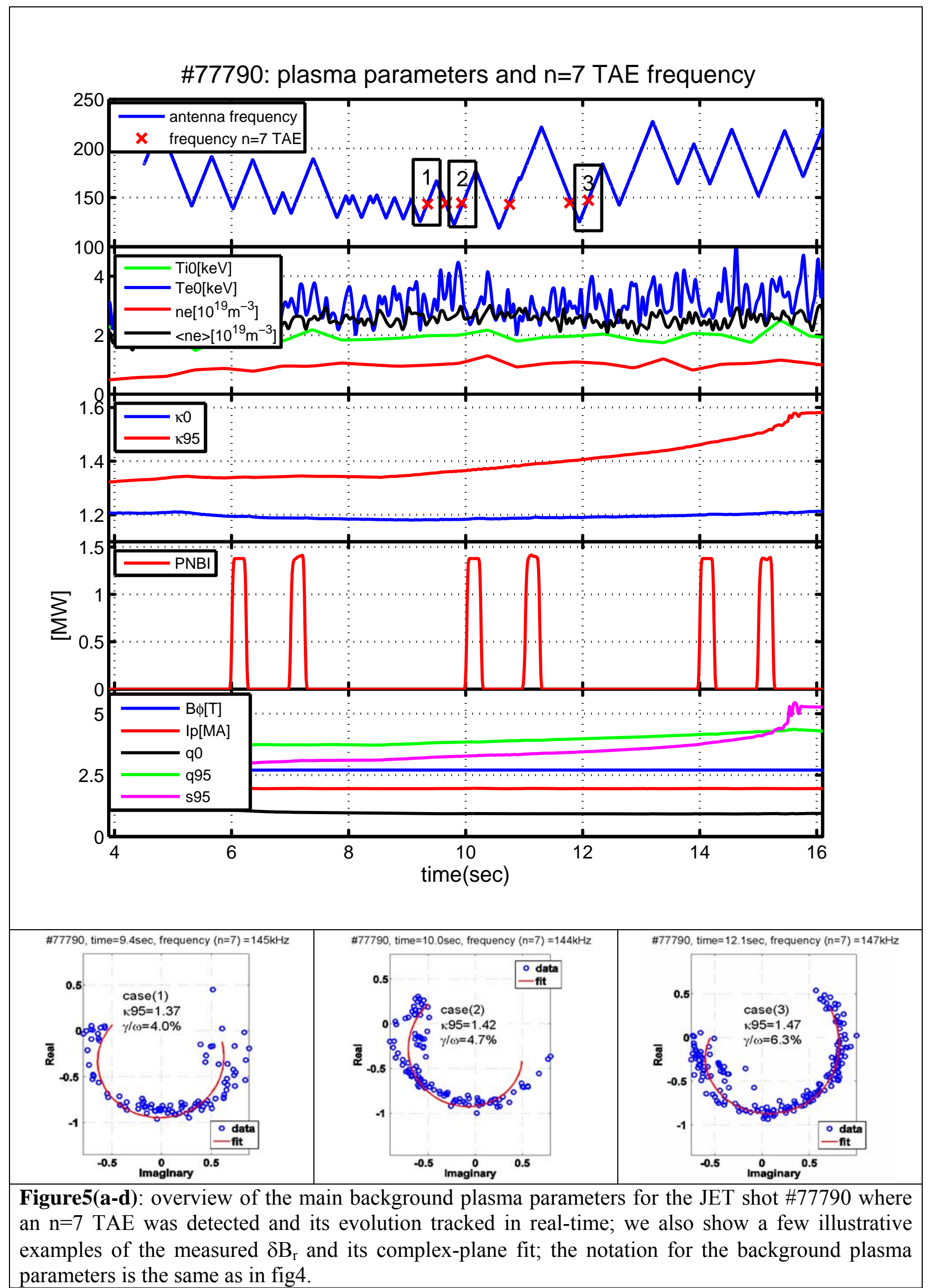

D.Testa, Figure5(a-d), paper NF-2010 post IAEA-TCM-EP-2009-ACCEPTED 


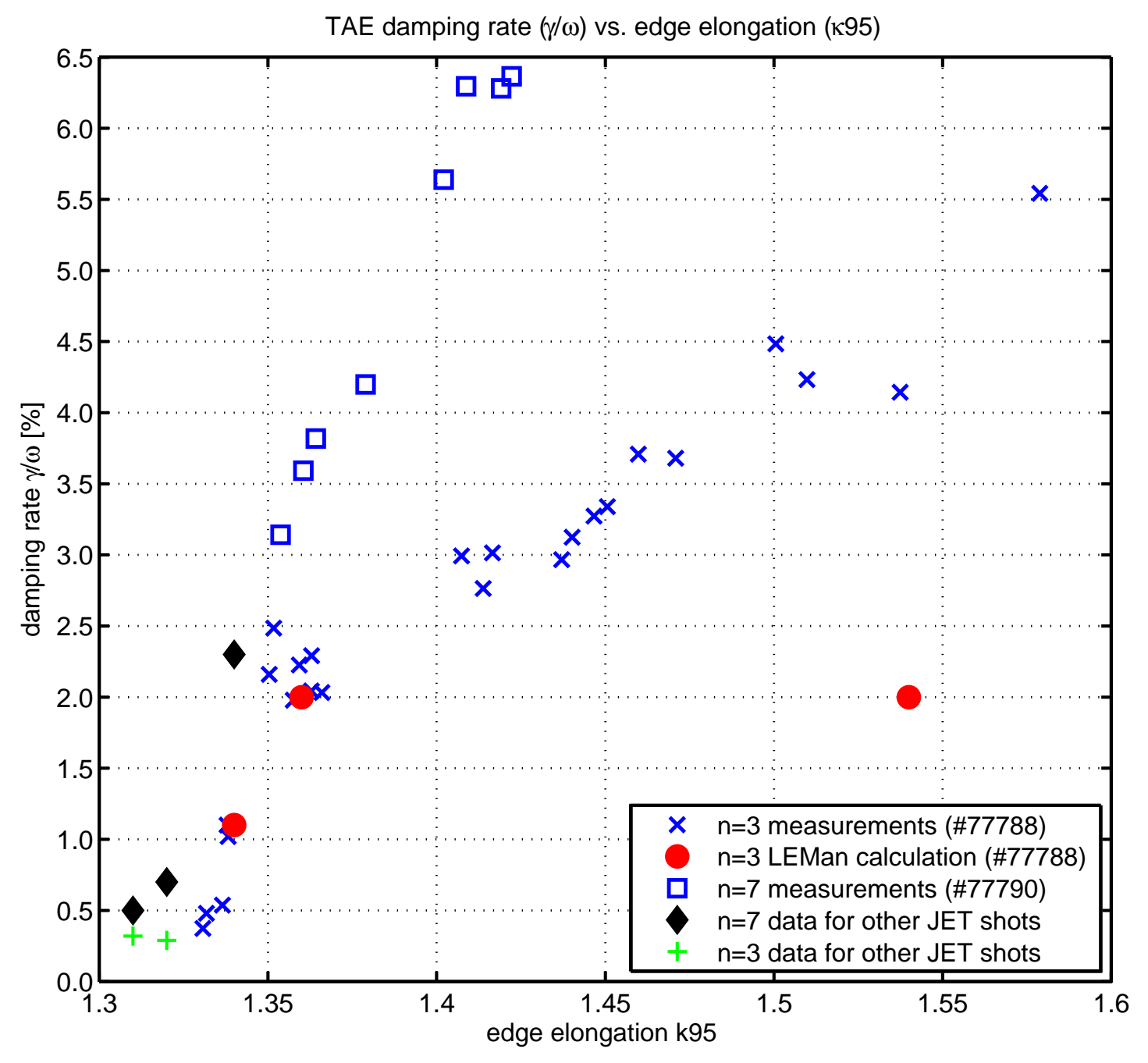

Figure6: the damping rate for the $n=3$ TAE (as measured and calculated using the LEMan code) and the $n=7$ TAE (only the measurements), as function of the edge elongation $\kappa_{95}$ in the JET discharges $\# 77788(n=3)$ and $\# 77790(n=7)$. For illustrative purposes, additional measurements are also plotted for the $n=3$ and the $n=n=7$ TAEs in other JET discharges, which are however not a sufficiently close match to the magnetic configuration and plasma profiles of \#77788 and \#77790: with this caveat in mind, the data indicate that a sharp increase in the damping rate occurs around $\kappa_{95} \sim 1.35$ for both the $\mathrm{n}=3$ and the $\mathrm{n}=7$ modes.

D.Testa, Figure6, paper NF-2010 post IAEA-TCM-EP-2009-ACCEPTED 


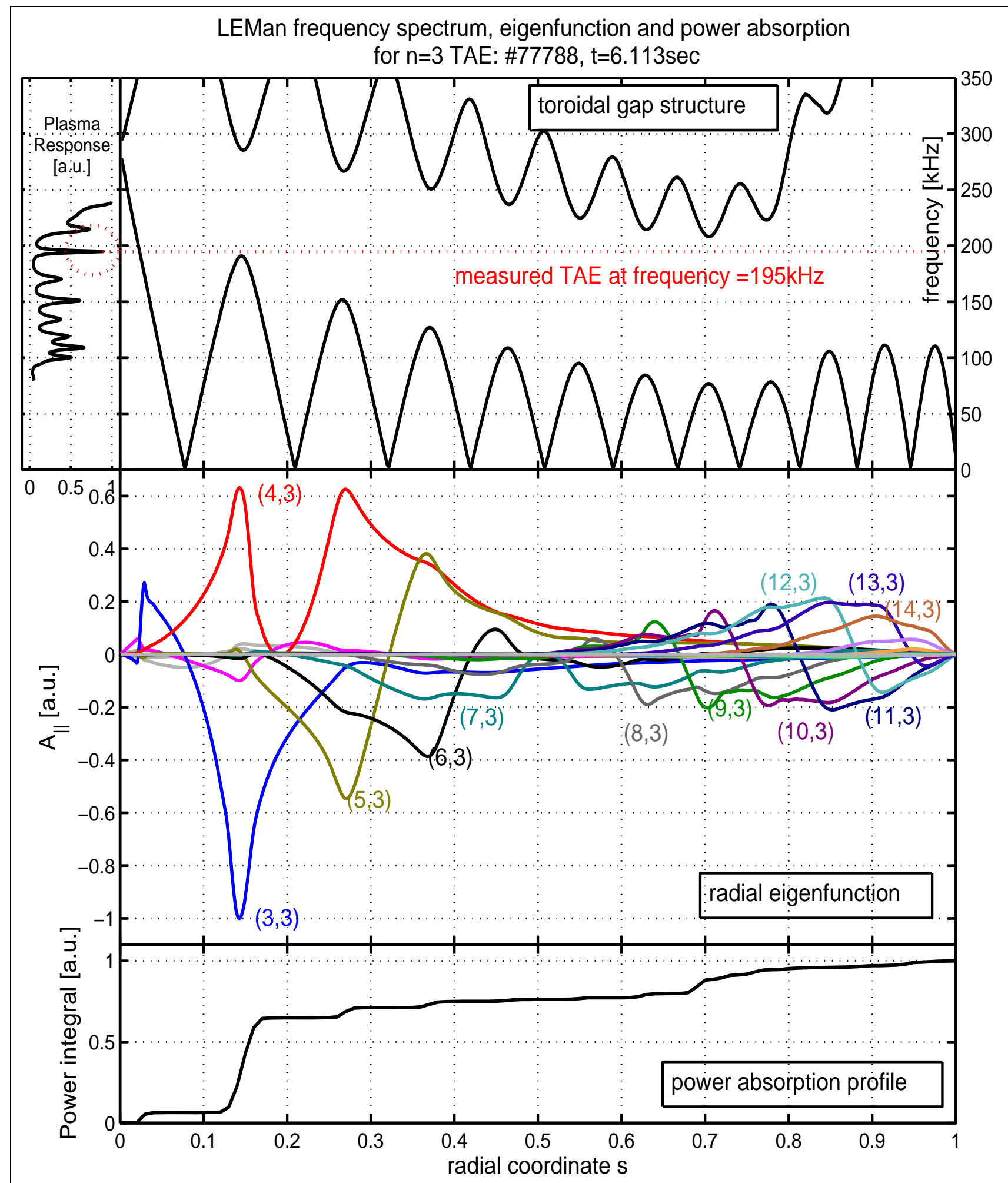

Figure7a: results of the LEMan simulation at $\mathrm{t}=6.113 \mathrm{sec}$ with the parameters on the axis $\mathrm{B}=2.7 \mathrm{~T}$, $\mathrm{n}_{\mathrm{e} 0}=2.2 \times 10^{19} \mathrm{~m}^{-3}$ and $\mathrm{T}_{\mathrm{e} 0}=\mathrm{T}_{\mathrm{i} 0}=2.6 \mathrm{keV}$. In the top frame, we show the frequency scan of the plasma response to the antenna drive (left sub-frame) and the Alfvén branches spectrum computed using the dispersion relation (right sub-frame); in the middle frame, we plot the Eigenfunction of the parallel component of the vector potential; in the bottom frame, we plot the integral of the power deposition profile for the calculated mode at $\mathrm{f}=195 \mathrm{kHz}$ (which corresponds to the measurement).

D.Testa, Figure7a, paper NF-2010 post IAEA-TCM-EP-2009-ACCEPTED 

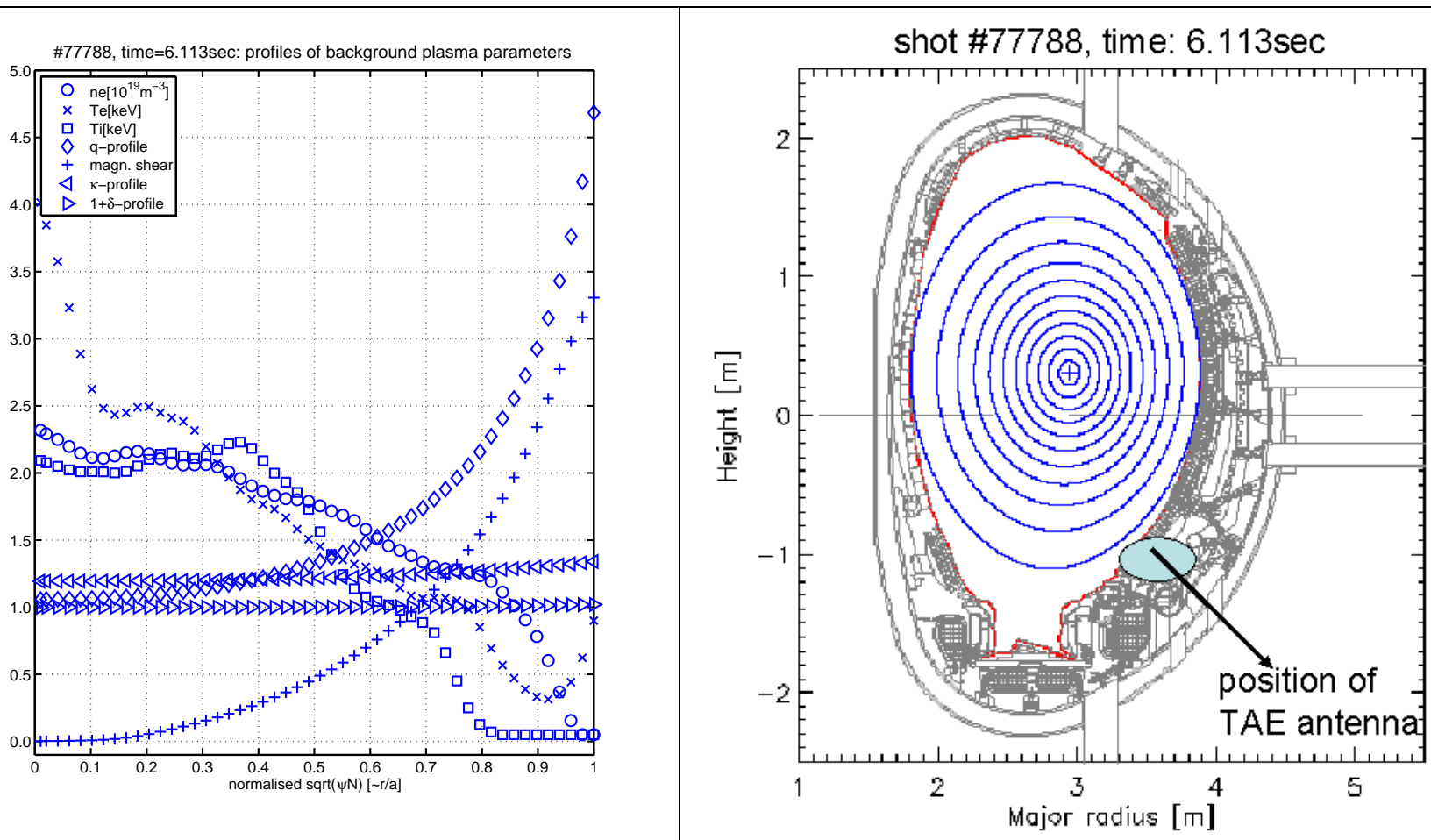

Figure7(b,c): the radial profiles of the main background plasma parameters (left frame: note that the ion temperature measurements are not reliable for $\mathrm{r} / \mathrm{a}>0.8$ and hence these data points are set to $\mathrm{T}_{\mathrm{i}}=0$ in this plot; similarly, there is a much larger error bar on the electron temperature measurements for $\mathrm{r} / \mathrm{a}>0.9$, hence the apparent increase in the $\mathrm{T}_{\mathrm{e}}$ data in this region is not entirely realistic) and the shape of the poloidal cross-section of the plasma (right frame) for $\# 77788$ at $t=6.113 \mathrm{sec}$; the position of the TAE antenna is also indicated for reference purposes.

D.Testa, Figure7(b,c), paper NF-2010 post IAEA-TCM-EP-2009-ACCEPTED 


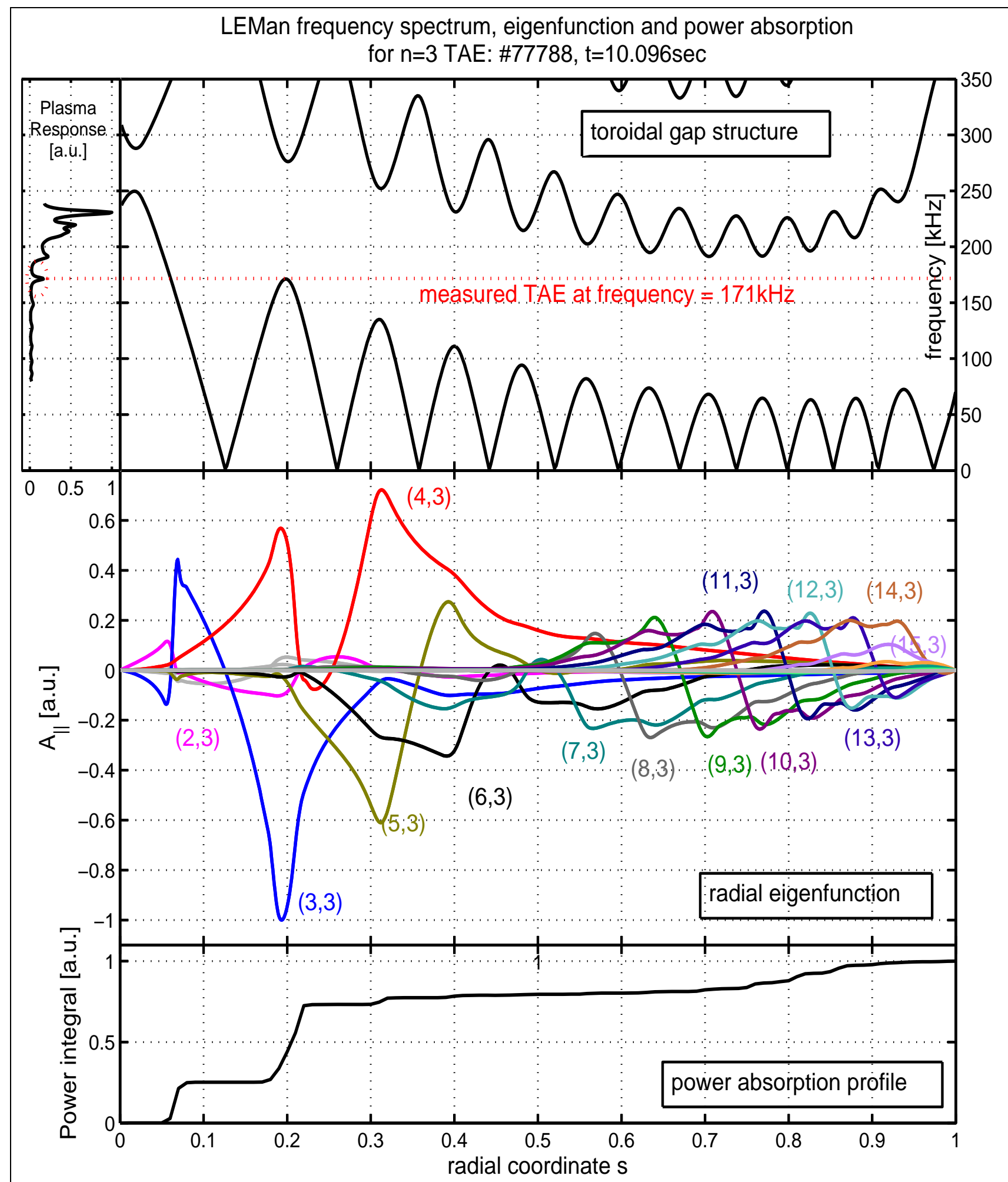

Figure8a: results of the LEMan simulation for the $n=3$ mode on $\# 77788$ at $t=10.096$ sec with the parameters on the axis $\mathrm{B}=2.7 \mathrm{~T}, \mathrm{n}_{\mathrm{e} 0}=2.6 \times 10^{19} \mathrm{~m}^{-3}$ and $\mathrm{T}_{\mathrm{e} 0}=\mathrm{T}_{\mathrm{i} 0}=2.4 \mathrm{keV}$. The convention for the frames is the same as in fig7a: the calculated mode at $\mathrm{f}=171 \mathrm{kHz}$ matches the measured frequency and is used for the calculation of the power absorption.

D.Testa, Figure8a, paper NF-2010 post IAEA-TCM-EP-2009-ACCEPTED 

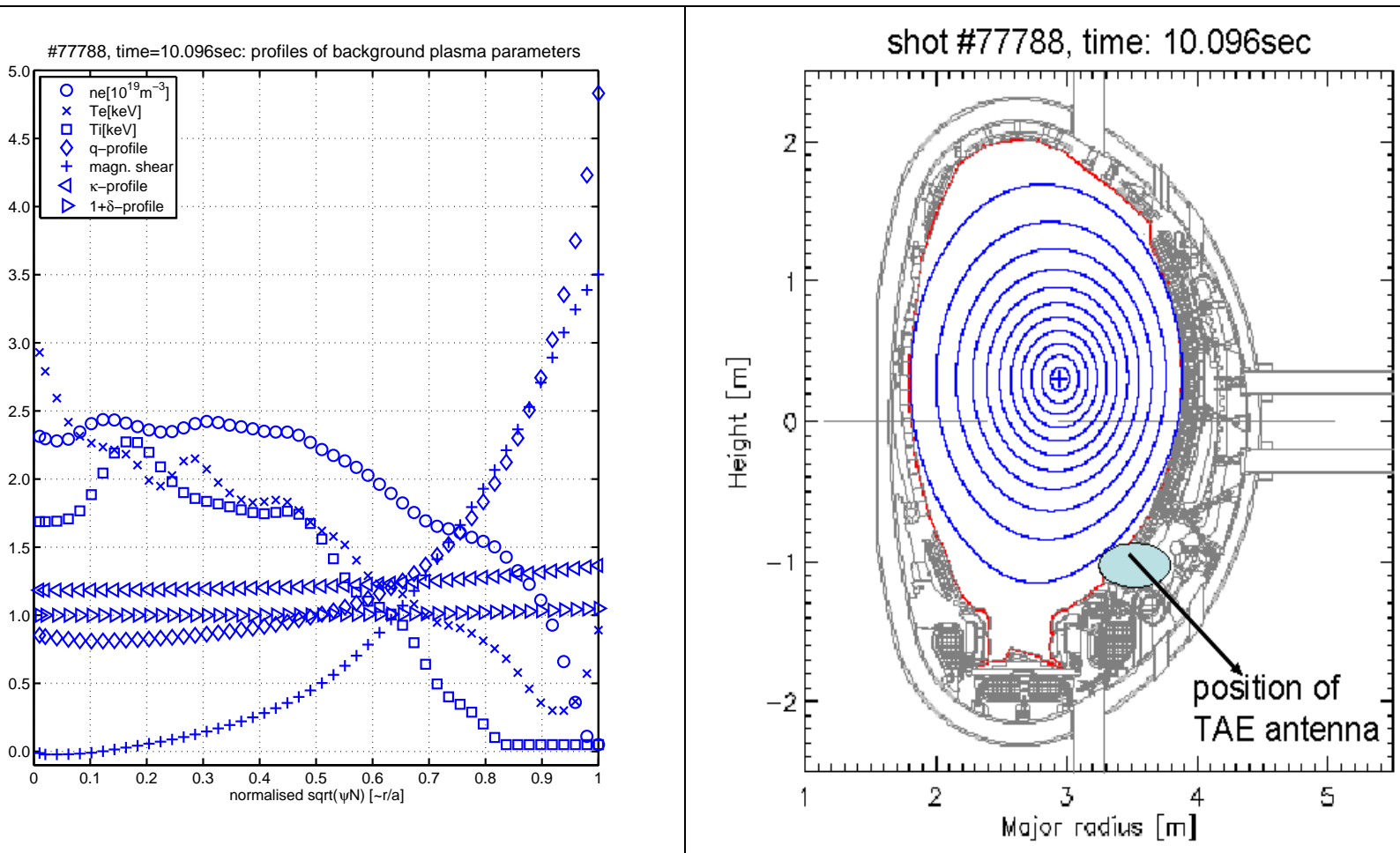

Figure8(b,c): the radial profiles of the main background plasma parameters (left frame: note that the same comments on the $\mathrm{Ti}$ and $\mathrm{T}_{\mathrm{e}}$ data made for fig $7 \mathrm{~b}$ apply here) and the shape of the poloidal crosssection of the plasma (right frame) for $\# 77788$ at $\mathrm{t}=10.096 \mathrm{sec}$; the position of the TAE antenna is also indicated for reference purposes.

D.Testa, Figure8(b,c), paper NF-2010 post IAEA-TCM-EP-2009-ACCEPTED 


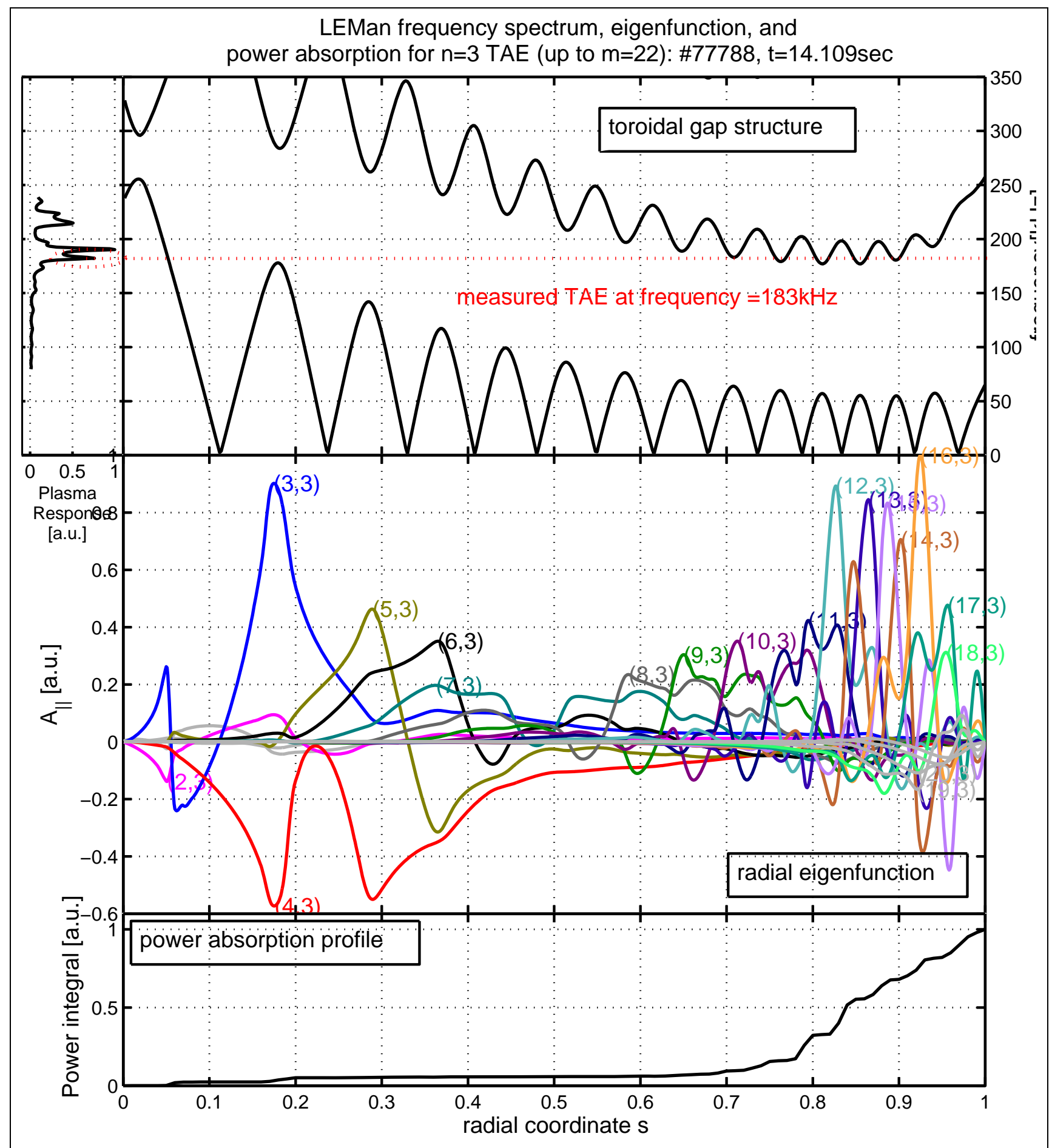

Figure9a: results of the LEMan simulation for the $n=3$ mode on $\# 77788$ at $t=14.109$ sec with the parameters on axis $\mathrm{B}=2.7 \mathrm{~T}, \mathrm{n}_{\mathrm{e} 0}=2.4 \times 10^{19} \mathrm{~m}^{-3}$ and $\mathrm{T}_{\mathrm{e} 0}=\mathrm{T}_{\mathrm{i} 0}=2.6 \mathrm{keV}$, using in this case all poloidal components $-5 \leq \mathrm{m} \leq 22$. The convention for the frames is as in fig $7 \mathrm{a}$ : the calculated mode at $\mathrm{f}=179 \mathrm{kHz}$ matches the measured frequency and is used for the calculation of the power absorption.

D.Testa, Figure9a, paper NF-2010 post IAEA-TCM-EP-2009-ACCEPTED 

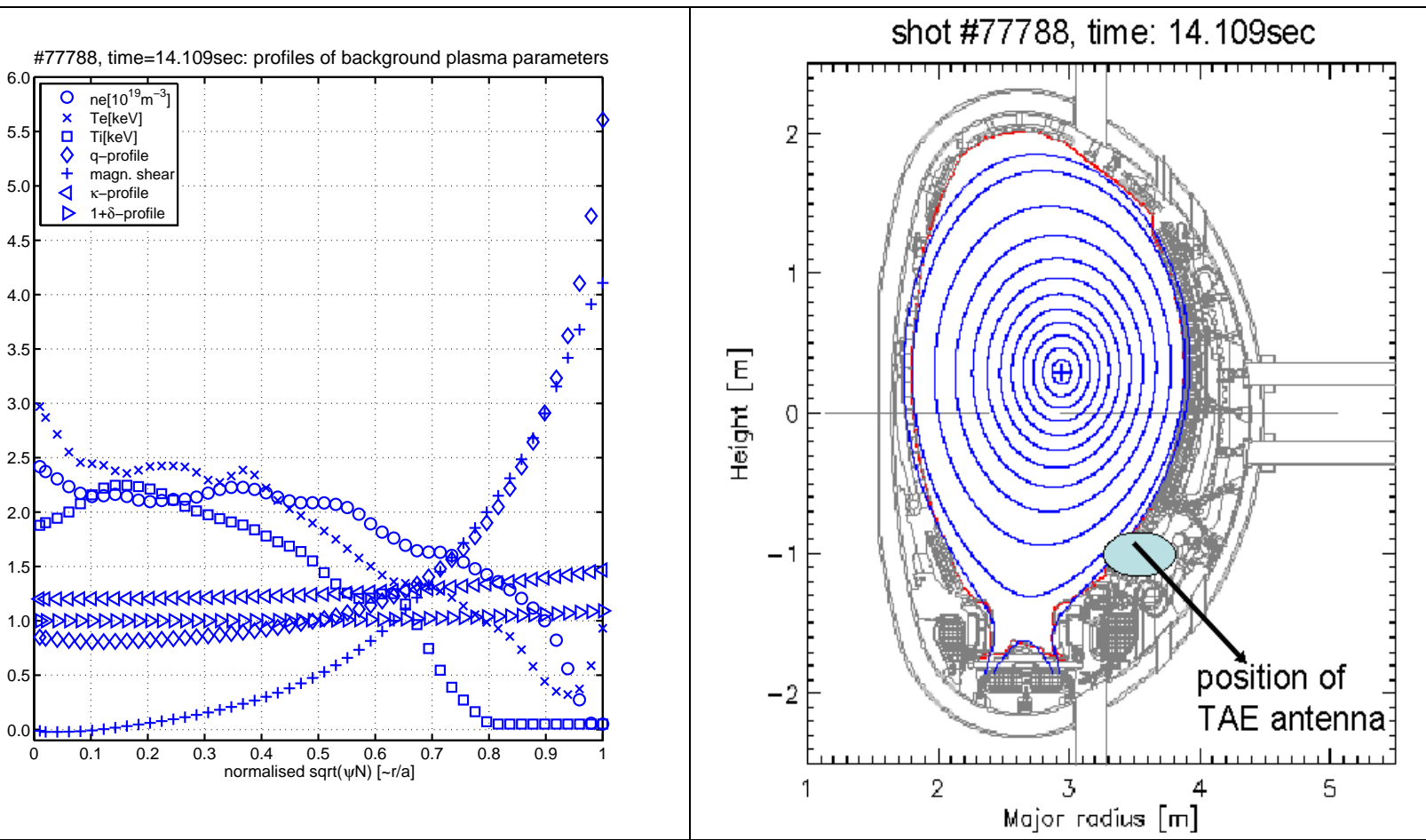

Figure9(b,c): the radial profiles of the main background plasma parameters (left frame: note that the same comments on the $\mathrm{Ti}$ and $\mathrm{T}_{\mathrm{e}}$ data made for fig $7 \mathrm{~b}$ apply here) and the shape of the poloidal cross-section of the plasma (right frame) for $\# 77788$ at $\mathrm{t}=14.109 \mathrm{sec}$; the position of the TAE antenna is also indicated for reference purposes.

D.Testa, Figure9(b,c), paper NF-2010 post IAEA-TCM-EP-2009-ACCEPTED 


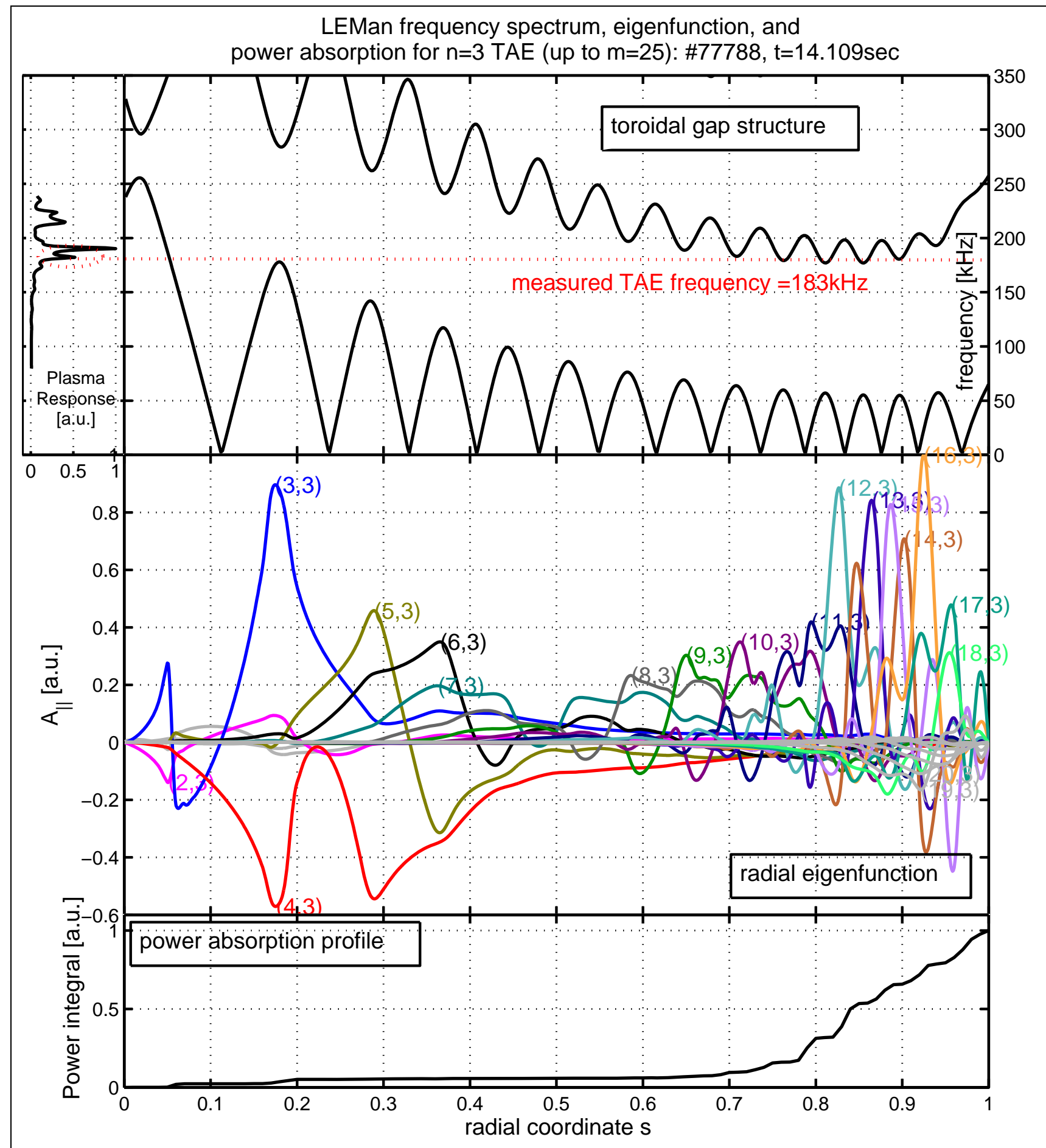

Figure10: results of the LEMan simulation at $\mathrm{t}=14.109 \mathrm{sec}$, using the same plasma data as in fig9, but now considering all poloidal components with $-5 \leq \mathrm{m} \leq 25$ : the calculated frequency spectrum, Eigenfunction and power absorption are very similar in these two cases.

D.Testa, Figure10, paper NF-2010 post IAEA-TCM-EP-2009-ACCEPTED 


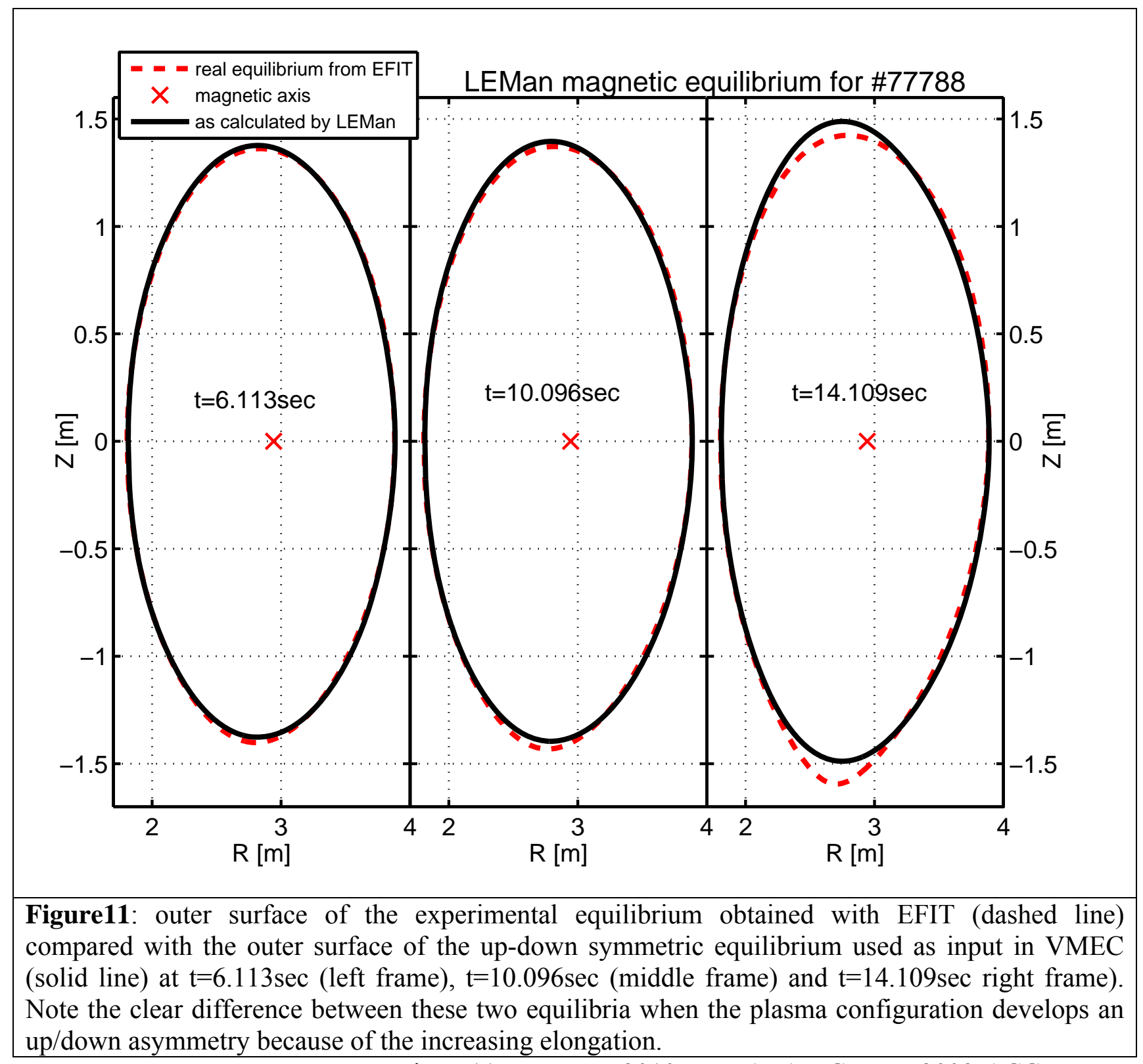

D.Testa, Figure11, paper NF-2010 post IAEA-TCM-EP-2009-ACCEPTED 